Published in final edited form as:

Nat Cell Biol. 2017 May ; 19(5): 504-517. doi:10.1038/ncb3514.

\title{
SWELL1 is a regulator of adipocyte size, insulin signaling and glucose homeostasis
}

\author{
Yanhui Zhang ${ }^{1}$, Litao Xie ${ }^{1}$, Susheel K. Gunasekar ${ }^{1}$, Dan Tong ${ }^{1}$, Anil Mishra ${ }^{1}$, William J. \\ Gibson $^{2}$, Chuansong Wang ${ }^{3}$, Trevor Fidler ${ }^{4}$, Brodie Marthaler ${ }^{1}$, Aloysius Klingelhutz ${ }^{5}$, E. \\ Dale Abel $^{4}$, Isaac Samuel ${ }^{6}$, Jessica K. Smith ${ }^{6}$, Lei Cao ${ }^{3}$, and Rajan Sah ${ }^{1,4,{ }^{*}}$ \\ ${ }^{1}$ Department of Internal Medicine, Division of Cardiovascular Medicine, University of lowa, Carver \\ College of Medicine, lowa City, IA, 52242 \\ ${ }^{2}$ Harvard Medical School, Boston, MA, 02115 \\ ${ }^{3}$ Department of Cancer Biology and Genetics, The Ohio State University, Columbus, Ohio \\ ${ }^{4}$ Fraternal Order of the Eagles Diabetes Research Center, lowa City, IA, 52242 \\ ${ }^{5}$ Department of Microbiology, University of lowa, Carver College of Medicine, lowa City, IA, 52242 \\ ${ }^{6}$ Department of Surgery, University of lowa, Carver College of Medicine, lowa City, IA, 52242
}

\section{SUMMARY}

\begin{abstract}
Adipocytes undergo considerable volumetric expansion in the setting of obesity. It has been proposed that such marked increases in adipocyte size may be sensed via adipocyte-autonomous mechanisms to mediate size-dependent intracellular signaling. Here, we show that SWELL1 (LRRC8a), a member of the Leucine Rich Repeat Containing protein family, is an essential component of a volume-sensitive ion channel (VRAC) in adipocytes. We find that SWELL1mediated VRAC is augmented in hypertrophic murine and human adipocytes in the setting of obesity. SWELL1 regulates adipocyte insulin-PI3K-AKT2-GLUT4 signaling, glucose uptake and lipid content via SWELL1 C-terminal leucine-rich repeat domain interactions with GRB2/Cav1. Silencing GRB2 in SWELL1 KO adipocytes rescues insulin-pAKT2 signaling. In vivo, shRNAmediated SWELL1 knock-down and adipose-targeted SWELL1 knock-out reduce adiposity and adipocyte size in obese mice while impairing systemic glycaemia and insulin-sensitivity. These studies identify SWELL1 as a cell-autonomous sensor of adipocyte size that regulates adipocyte growth, insulin sensitivity and glucose tolerance.
\end{abstract}

\footnotetext{
Users may view, print, copy, and download text and data-mine the content in such documents, for the purposes of academic research, subject always to the full Conditions of use: http://www.nature.com/authors/editorial_policies/license.html\#terms

"Corresponding Author: Dr. Rajan Sah, 169 Newton Rd. PBDB 4334, Iowa City, Iowa 52242, Phone: 319-353-5660 rajansah@uiowa.edu. AUTHOR CONTRIBUTIONS

Conceptualization, R.S.; Methodology, Y.Z., L.X., S.G., D.T., W.J.G., T.F., R.S.; Formal Analysis, R.S., Y.Z., L.X., S.G, D.T., A.M., W.J.G., T.F.; Investigation, R.S., Y.Z., L.X., S.G, D.T., T.F., B.M., A.M.; Resources, C.W., L.C., E.D.A., J.S., I.S.; Writing - Original Draft, R.S., Writing - Review \& Editing, R.S., Y.Z., L.X., S.G., D.T. E.D.A.; Visualization, R.S., Y.Z., L.X., S.G; Supervision, R.S.; Funding Acquisition, R.S.
} 


\section{INTRODUCTION}

The adipocyte maximizes energy storage by forming a large lipid droplet, separated from the plasma membrane by a thin rim of cytoplasm, while maintaining a tremendous capacity for volumetric expansion - increasing $>30$-fold in the setting of obesity ${ }^{1}$. This lipid droplet growth augments adipocyte stiffness, thereby reducing membrane compliance ${ }^{2}$ and increasing membrane tension, which in turn has been shown to activate intracellular signaling pathways ${ }^{3,4}$, implying that adipocytes may be mechano-sensitive ${ }^{4}$. Moreover, adipocyte size in obesity has been correlated with the severity of linked diseases such as diabetes and insulin resistance ${ }^{5,6}$, suggesting that increases in adipocyte volume ${ }^{1}$ or membrane tension ${ }^{7}$ may alter intracellular signaling. Others propose that caveolae enable expanding adipocytes to auto-regulate lipid content based on mechanical lipid dropletplasma membrane interactions ${ }^{8}$ and tune insulin signaling in response to adipocyte swelling ${ }^{9}$. However, the molecular identity a putative adipocyte volume sensor remains a mystery.

Ion channels are membrane proteins that can signal in response to membrane-stretch, and accordingly provide plausible candidates for adipocyte membrane stretch-sensors that bridge membrane tension with intracellular signaling. There are a number of candidate stretch/ mechano-sensitive ion channels in mammalian cells including TRPM $7{ }^{10,11}$, TRPV $2^{12}$, TRPV4 ${ }^{13}$, TRPC6 ${ }^{14}$ and Piezo-1/Piezo- ${ }^{15}$. Many of these ion channels are expressed in adipocytes ${ }^{16}$, and have signaling roles important for adipogenesis ${ }^{16}$, fatty acid sensing ${ }^{17}$, oxidative metabolism, inflammation and energy homeostasis ${ }^{18}$.

In this study, we explored swell-activated ion channel signaling in adipocytes by applying the patch-clamp technique to freshly isolated, mature murine and human adipocytes. Using this approach, we identified a prominent swell-activated chloride current in adipocytes, characteristic of the volume-regulated anion current (VRAC), and discovered that the gene LRRC8a, a member of the Leucine Rich Repeat (LRR) Containing proteins ${ }^{19,} 20$ (SWELL1), is required for adipocyte VRAC. Interestingly, Kumar et al. ${ }^{21}$, unaware of the volume-sensitive ion channel function of SWELL1 (LRRC8a), recently showed that it can signal via phosphoinositide 3-kinase (PI3K)/AKT in lymphocytes, a pathway known to be important for lipid and glucose homeostasis in adipocytes ${ }^{22,23}$. On the background of these recent findings we hypothesized that SWELL1 may participate in sensing adipocyte volume during physiological or pathophysiological adipocyte expansion and engage insulin-PI3KAKT signaling - thereby coupling adipocyte growth with insulin signaling. Herein, we link the volume-sensitive SWELL1 molecule to adipocyte insulin signaling and growth; and propose a model in which SWELL1 activates in response to adipocyte expansion, and tunes insulin-mediated activation of growth and glucose import pathways.

\section{Adipocyte patch clamp reveals prominent swell-activated current in murine and human adipocytes}

To identify a putative swell-activated ionic current in adipocytes we performed patch-clamp recordings on mature murine adipocytes freshly isolated from inguinal white adipose tissue (iWAT). We measured ionic currents in voltage-clamp mode while simultaneously swelling the adipocyte by applying positive pressure $(\sim 3-5 \mathrm{mmHg}$, whole-cell configuration) via the 
patch-pipette (Fig. 1a-Swelled). After a few minutes, we observed activation of a large swell-activated current (Fig. 1b\&c). This current is outward rectifying, characterized by larger outward current at positive voltages compared to inward currents at negative voltages during sequential voltage ramps (Fig. 1b\&c) or voltage steps (Fig. 1d), with the current amplitude proportional to the extent of adipocyte swelling (Fig. 1e-g). This current reverses at $-11.7 \pm 2.5 \mathrm{mV}$, which is near the reversal potential of chloride under our conditions $(-11.5 \mathrm{mV}$, junction potential corrected). Application of low chloride extracellular solution largely abolished the outward component of this current and shifted the current reversal towards $0 \mathrm{mV}$ (Fig. 1h), suggesting a predominant chloride conductance. Finally, this swellactivated adipocyte current is blocked by 4-[(2-Butyl-6,7-dichloro-2-cyclopentyl-2,3dihydro-1-oxo-1 $H$-inden-5-yl)oxy]butanoic acid (DCPIB), a selective blocker of the volume-regulated anion current (VRAC) or volume-sensitive outwardly rectifying chloride current (VSOR) (Fig. 1b-c). We also confirmed this swell-activated current to be present in the murine 3T3-F442A (Fig. 1i) and human adipocyte cell lines (Fig. 1j, NPAD) ${ }^{24}$ upon hypotonic swelling, and this current is fully inhibited by DCPIB. Collectively, these data suggest that this swell-activated adipocyte chloride current is VRAC or VSOR, as identified previously in numerous other cell types ${ }^{25,26}$, including adipocytes ${ }^{27}$.

\section{SWELL1/LRRC8a is required for swell-activated VRAC in adipocytes}

As SWELL1-LRRC8-complexes have been recently shown to form the ion channel responsible for VRAC in several common cell lines ${ }^{19,} 20,28$, we next asked whether SWELL1 was required for adipocyte VRAC. We generated an adenovirus expressing a short-hairpin RNA directed to SWELL1 mRNA (Ad-shSWELL1-mCherry) to knock-down SWELL1 in both mature, primary adipocytes and in adipocyte cell lines, while a scrambled shRNA served as a control (Ad-shSCR-mCherry). We confirmed robust knock-down (KD) of SWELL1 mRNA (Supplementary Fig. 1a) and protein (Supplementary Fig. 1b) in cultured human pre-adipocytes upon adenoviral shRNA transduction. To knock-down SWELL1 in mature, primary murine adipocytes, we injected Ad-shSWELL1/shSCRmCherry directly into iWAT of mice and then isolated and recorded from patch-clamped mCherry positive adipocytes 5 days later (Fig. 2a). ShRNA-mediated SWELL1 knock-down nearly abolished all swell-activated VRAC in murine adipocytes as compared to the scrambled shRNA control (Fig. 2b\&c), in addition to VRAC in both murine 3T3-F442A adipocytes (Supplementary Fig. 1c\&d) and in a human adipocyte cell line (Supplementary Fig. 1e\&f).

As a complementary approach, we used CRISPR/cas9 gene editing ${ }^{29}$ to disrupt $S W E L L 1$ using either a single guide RNA (gRNA; Supplementary Fig. 2a) or double gRNA (Supplementary Fig. 2b\&c) approach in the 3T3-F442A adipocyte line. From two independent $S W E L L 1 \mathrm{CRISPR} /$ cas9 knock-out (KO) adipocyte cell lines we find that VRAC is entirely abolished (Fig. 2d\&e) upon $S W E L L 1$ gene disruption, and this is associated with complete loss of SWELL1 protein (Fig. 2f).

To achieve conditional ablation of $S W E L L 1$, we generated mice with flanking loxP sites around $S W E L L 1$ Exon 3 by applying a CRISPR/cas9 knock-in approach ${ }^{30}$ (Supplementary Fig. 2d). Cultured tail-tip fibroblasts from $S W E L L 1^{f l}$ mice transduced with adenoviral-Cre- 
mCherry (Ad-Cre-mCherry) show excision of SWELL1 Exon 3 (Supplementary Fig. 2e). Primary adipocytes isolated and cultured from the stromal vascular fraction (SVF; Supplementary Fig. 2f) also show specific $S W E L L 1$ mRNA ablation when transduced (>90\% efficient) with Ad-Cre-mCherry (Supplementary Fig. $2 \mathrm{~g} \& \mathrm{~h}$ ), and this is associated with ablation of VRAC (Supplementary Fig. 2i-k). Next, we measured VRAC in mature murine adipocytes isolated from iWAT of $S W E L L I^{\mathrm{fl}}$ mice 5-7 days after direct injection with either Ad-mCherry or Ad-Cre-mCherry (Fig. 2g). We find that $S W E L L 1$ deletion (AdCre-mCherry) entirely ablates swell-induced VRAC in mature adipocytes (Figure $2 \mathrm{~h}-\mathrm{j}$ ). Collectively, these data show that SWELL1/LRRC8a is required for swell-induced VRAC currents in both primary adipocytes and adipocyte cell lines.

\section{SWELL1-mediated VRAC is increased in adipocytes of obese mice and humans}

As adipocyte hypertrophy is the relevant physiological stimulus encountered in vivo, we asked whether lipid droplet expansion in the setting of obesity could activate SWELL1mediated VRAC at the adipocyte plasma membrane. We isolated mature adipocytes from iWAT of lean (normal chow: NC, 10-12 weeks) and obese (high fat diet, HFD, 10-12 weeks; Supplementary Fig. 3a) mice and measured VRAC using the perforated-patch configuration of the patch-clamp technique (Fig. 3a, see Methods) pre- and post hypotonic swelling. Similar to positive-pressure swelling, we discovered that a minimal amount of adipocyte swelling is required ( 1-2\% increase in diameter) to activate robust SWELL1mediated currents (Fig. 3a\&b; i to ii) - easily within the physiological range of adipocyte expansion/contraction occurring during feeding/fasting. As expected, adipocytes isolated from obese mice are hypertrophic compared to lean mice based on cell size (Fig. 3c) and cell capacitance (Fig. 3d), the latter providing a measure of adipocyte surface area. SWELL1mediated current densities in hypertrophic adipocytes are significantly increased compared to adipocytes of lean mice, both upon adipocyte swelling (Fig. 3e-g) and, importantly, prior to swelling (Fig. 3e\&h, pre-stimulation). To determine whether these increases in SWELL1mediated currents measured in hypertrophic adipocytes result from SWELL1-LRRC8 channel activation versus augmented SWELL1-LRRC8 channel expression, we performed qPCR to measure relative mRNA expression levels of SWELL1/LRRC8a, LRRC8b, LRRC8c, LRRC8d and LRRC8e in adipocytes from lean compared to obese mice (Supplementary Fig. 3b), and found a non-statistically significant trend toward increasing SWELL1 (2.2-fold; $\mathrm{p}=0.07$ ) expression, with significant increases in LRRC8b (3-fold, $\mathrm{p}<$ $0.05)$ and LRRC8d (2.9-fold, $\mathrm{p}<0.01)$. Therefore, it is possible that augmented adipocyte VRAC currents in obesity occur due to SWELL1-LRRC8 channel activation by adipocyte hypertrophy, in addition to coordinated increases in SWELL1/LRRC8a, LRRC8b and LRRC8d expression.

We next asked whether VRAC is also activated in hypertrophic adipocytes of obese humans and patch-clamped mature adipocytes freshly dissociated from visceral fat harvested from obese, BMI > 30, bariatric surgery patients compared to leaner non-bariatric patients, BMI < 30 (see Supplementary Fig. 3c). As in mice, there is a significant, though less marked, increase in adipocyte size (Figure 3i) and adipocyte capacitance (Figure 3j) in obese compared to leaner non-bariatric patients. Similarly, VRAC is augmented upon swelling in adipocytes from obese compared to leaner patients (Figure 3k-m), as is basal or "pre- 
activated" VRAC inward current (Fig. 3k\&n, pre-stimulation). Taken together, these data strongly suggest that SWELL1-mediated VRAC is augmented in adipocytes as they hypertrophy in obese mice and humans.

\section{SWELL1 regulates lipid content and glucose metabolism in 3T3-F442A adipocytes}

To obtain unbiased insight into putative SWELL1-dependent molecular pathways impacting adipocyte function we performed genome-wide RNA sequencing (RNA Seq) of wild-type (WT) and CRISPR/cas9 mediated SWELL1 KO adipocytes. This revealed significant differences in RNA transcriptional profile with 9090 differentially expressed genes based on a false-discovery rate q-value of 0.25 (Supplementary Fig. 4a; Supplementary Table 1). $\underline{G}$ ene $\underline{S}$ et $\underline{E}$ richment $\underline{A}$ nalysis (GSEA) of the RNA sequencing data highlights pathways related to hypoxia, adipogenesis, glucose metabolism and insulin signaling (Supplementary Fig. $4 \mathrm{~b}$ and Supplementary Table 2). Consistent with a requirement for $S W E L L 1$ in adipogenesis, both $S W E L L 1$ KO adipocytes (Fig. 4 a) and cultured $S W E L L 1$ KO primary adipocytes (Fig. 4b) develop less intracellular lipid than WT as assessed by AdipoRed fluorescence imaging (under high glucose culture conditions, $25 \mathrm{mM}$ ). Switching to low glucose media $(5 \mathrm{mM})$ abolishes this difference in intracellular lipid content between WT and $S W E L L 1 \mathrm{KO}$ adipocytes (Supplementary Fig. 4c). These data suggest that impaired glucose uptake, and consequently diminished de novo lipogenesis, underlies the reduction in lipid content in SWELL1 KO adipocytes relative to WT under high glucose culture conditions.

We next measured basal and insulin-stimulated glucose uptake in WT and SWELL1 KO adipocytes using radioactively labeled 2-deoxy-D-glucose. WT adipocytes exhibit the expected increase in glucose uptake upon insulin-stimulation ${ }^{31}$, while this effect is abrogated in SWELL1 KO adipocytes (Fig. 4c). Transmission electron microscopy (TEM) images of $S W E L L 1 \mathrm{KO}$ adipocytes reveal a significant reduction in glycogen content compared to WT adipocytes under conditions of chronic insulin stimulation (i.e. differentiation media, 860 nM) (Fig. 4d, Supplementary Fig. 4d). Taken together, these data suggest that SWELL1 is required for both glucose uptake/metabolism and lipogenesis in adipocytes.

\section{SWELL1 regulates insulin-PI3K-AKT signaling, GLUT4 translocation, GSK3 $\beta$ and FOXO1}

As both glucose uptake and lipogenesis require intact insulin-phosphoinositol 3-kinaseAKT2 (insulin-PI3K-AKT2) signaling in adipocytes ${ }^{22,32}$, we next examined the integrity of this pathway in SWELL1 KO adipocytes. SWELL1 deletion in both SWELL1 KO 3T3F442A adipocytes (Fig. 4e) and $S W E L L 1$ KO primary adipocytes (Fig. 4f) significantly reduced insulin-stimulated pAKT2 (pSerine474) when compared to their respective WT adipocytes, whereas pAKT1 (pSerine473) levels remained constitutively increased. To determine whether SWELL1 silencing in mature adipocytes in vivo also disrupts insulinPI3K-AKT signaling we examined insulin-stimulated pAKT2 and pAKT1 in iWAT of mice 7 days after direct iWAT injection with Ad-shSCR-mCherry/Ad-shSWELL1-mCherry. Paralleling the in vitro data, iWAT SWELL1 knock-down significantly dampened insulinpAKT2 signaling without influencing insulin-mediated phosphorylation of AKT1 (Fig. 4g). 
As insulin-PI3K-AKT2 stimulates glucose uptake via GLUT4 plasma membrane translocation, we directly measured insulin-stimulated GLUT4 translocation, using a surface biotinylation, and then blotted for GLUT4 in the plasma membrane (PM) versus cytosol (C). We find that insulin-stimulated GLUT4 PM translocation is reduced in SWELL1 KO adipocytes (Fig. 4h). As it is well established that phosphorylation-mediated inhibition of the RabGAP AS160 (pThreonine642) is required for insulin-stimulated GLUT4 vesicle docking and fusion with the $\mathrm{PM}^{33,34}$, and that this phosphorylation event is AKT2-specific and AKT1-independent ${ }^{32}$ we next examined insulin-stimulated pAS160/AS160. We find that pAS160/AS160 is reduced in SWELL1 KO adipocytes compared to WT (Fig. 4i), consistent with the observed reduction in insulin-PI3K-pAKT2-GLUT4 signaling.

Insulin-PI3K-AKT-mediated phosphorylation of Glycogen Synthase Kinase-3 $\beta$ (pGSK3 $\beta$ ) inhibits GSK3 $\beta$ activity and subsequently dis-inhibits glycogen synthase, allowing glucose to be stored as glycogen ${ }^{35}$. We find that insulin-stimulated pGSK3 $\beta /$ GSK3 $\beta$ and pGSK3 $\beta / \beta$ actin (pSerine9) are reduced in $S W E L L 1$ KO adipocytes (Fig. 4j), providing a molecular mechanism for the reduction in glycogen granules observed on EM (Fig. 4d). Thus, SWELL1-mediated pAKT2 signaling appears to coordinately regulate both glucose import via GLUT4 translocation and glycogen synthesis via GSK3 $\beta$. We next examined the AKTsubstrate FOXO1, a transcription factor that suppresses adipocyte differentiation when nuclear localized, but upon insulin-AKT-mediated phosphorylation pFOXO1 becomes excluded from the nucleus - thereby releasing adipocyte differentiation programs ${ }^{36}$. We find that insulin-AKT-mediated pFOXO1/FOXO1 and pFOXO1/ $\beta$-actin (pThreonine24), are enhanced in SWELL1 KO adipocytes (Fig. 4j), in a manner similar to pAKT1. Taken together, these data show that SWELL1 is required for insulin-PI3K-AKT2 activity and downstream signaling in adipocytes and suggest that SWELL1 may connect volume of the expanding adipocyte with "tuning" of insulin-PI3K-AKT2 signaling.

\section{SWELL1 regulates insulin-pAKT2 signaling via GRB2-dependent interactions}

In lymphocytes, SWELL1 connects with PI3K signaling via a Growth Factor Receptorbound 2 (GRB2) interaction ${ }^{21}$. GRB2 is linked to insulin receptor (IR) signaling via a direct interaction with insulin-receptor substrate (IRS) $1 / 2^{37}$ and is thought to modulate insulin signaling. We confirmed that GRB2 co-immuno-precipitates (IP) with IR in both 3T3F442A adipocytes (Supplementary Fig. 5a) and HEK cells (Supplementary Fig. 5b); and that GRB2 co-IPs with IRS1 (using an epitope-tagged IRS1 construct: Myc-TEV-HA-IRS1) in HEK cells (Supplementary Fig. 5c). Next, we tested for an endogenous GRB2-SWELL1 interaction in adipocytes. We find that GRB2 co-IPs with SWELL1 in WT but not SWELL1 $\mathrm{KO}$ adipocytes, and that insulin-stimulation reduces this $S W E L L 1-\mathrm{GRB} 2$ interaction (Fig. 5a), suggesting a dynamic, insulin-dependent SWELL1-GRB2 interaction (see SWELL1/ GRB2 densitometry).

GRB2 has also been reported to associate with caveolin-1 (Cav1) ${ }^{38}$. Cav1 is enriched in adipocytes ${ }^{39}$, thought to form insulin signaling microdomains ${ }^{40}$, is required for normal adipocyte insulin signaling ${ }^{41}$ and has been described as a cellular mechanosensor ${ }^{8,42}$ that regulates PI3K-AKT signaling ${ }^{43}$. Moreover, there are reports that Cav1 regulates VRAC ${ }^{44}$, further suggesting a putative SWELL1-Cav1 molecular interaction. We find that Cav1 co- 
IPs with GRB2 in adipocytes (Fig. 5a), and SWELL1 also co-IPs with Cav1 in WT but not in SWELL1 KO adipocytes (Fig. 5b). These data suggest that SWELL1 resides in an insulin signaling macromolecular complex that includes Cav1, GRB2, IRS-1, and IR.

It has been proposed that the SWELL1 $\underline{\boldsymbol{L}}$ eucine- $\underline{\boldsymbol{R}} \mathrm{ich} \underline{\boldsymbol{R}}$ epeat $\underline{\boldsymbol{D}}$ omain (LRRD) may provide docking surfaces for protein-protein interactions for signaling to various protein-kinases ${ }^{45}$. To determine if SWELL1 LRRD is required for GRB2 binding, we expressed wild-type $S W E L L 1$ (Fig. 5c), and the $S W E L L 1$ mutant, $S W E L L 1^{491 /+35}$ (Fig. 5d), in HEK cells and attempted to pull-down these $S W E L L 1$ proteins with endogenous GRB2. SWELL $1^{\Delta 91 /+35}$ is a mutation which replaces $91 \mathrm{C}$-terminal LRRD amino acids with 35 amino acids derived from the neighboring intronic sequence ${ }^{46}$ (Fig. 5d); this partially disrupts the LRRD, and inactivates SWELL1 activity ${ }^{20}$. When WT $S W E L L 1$ and $S W E L L 1^{\triangle 91 /+35}$ are transiently expressed at equal levels in HEK cells $\left(S W E L L 1 / \beta\right.$-actin $=1.38 ; S W E L L 1^{\Delta 91 /+35} / \beta$-actin $=$ 1.41 ; Fig. 5e) we find that $S W E L L 1^{\triangle 91 /+35}$ co-IP's with GRB2 less efficiently $\left(S W E L L 1^{\Delta 91 /+35 / G R B 2}=0.07\right)$ than WT SWELL1 $(S W E L L 1 /$ GRB2 $=0.31)($ Fig. $5 \mathrm{e})$, suggesting that the SWELL1-LRRD is required for GRB2 binding.

We next asked how a SWELL1-GRB2 complex may regulate insulin signaling. It is noteworthy that GRB2 loss of function enhances insulin-PI3K-AKT and MAP kinase signaling in cultured hepatocytes ${ }^{47}$ and in $\mathrm{C} 2 \mathrm{C} 12$ myoblasts ${ }^{48}$; and also augments insulin signaling and glucose tolerance in vivo ${ }^{48}$, suggesting that GRB2 is a negative regulator of insulin signaling. We find that insulin-stimulation reduces both SWELL1-GRB2 and Cav1GRB2, but not SWELL1-Cav1 interactions in WT adipocytes (Fig. 5a\&b), suggesting that insulin-stimulation induces GRB2 dissociation from the insulin-signaling complex, thereby dis-inhibiting downstream insulin signaling. To examine whether the defect in insulinpAKT2 signaling observed upon SWELL1 KO is due to unrestrained GRB2-mediated inhibition we generated stable WT/GRB2 KD and SWELL1 KO/GRB2 KD adipocytes using a lentiviral shRNA to GRB2. Similar to prior reports in hepatocytes and myoblasts, we observe enhanced insulin-pAKT2 signaling in WT GRB2-deficient adipocytes (Fig. 5f, g \& h), even under non-insulin stimulated conditions. Importantly, GRB2 KD in SWELL1 KO adipocytes rescues the impairment in insulin-pAKT2 activation, confirming that suppression of insulin-pAKT2 signaling upon SWELL1 ablation is GRB2-dependent (Fig. 5f, i \& j). Overall, these data suggest that SWELL1 modulates insulin-PI3K-AKT2 signaling by titrating GRB2-inhibition of a Cav1-IRS1-IR signaling complex via a C-terminal LRRDmediated interaction with GRB2.

\section{SWELL1 knock-down reduces adipocyte size, adiposity and impairs glucose tolerance in obese mice}

Based on our findings that SWELL1 is required for insulin-PI3K-AKT2 signaling and that SWELL1 is activated in hypertrophic adipocytes in the context of obesity, we asked whether $S W E L L 1$ knock-down can influence adipocyte size, adiposity and glucose tolerance in obese mice. We tested the acute effects of SWELL1 knock-down on adipocyte size, by injecting iWAT of obese mice (HFD, 10-12 weeks) with either Ad-shSCR-mCherry or Adsh SWELL1-mCherry, and after 12 days, isolating and measuring the transduced adipocyte. We find that SWELL1-depleted adipocytes are 28\% smaller than control adipocytes (Fig. 
6a\&b) with a leftward shifted size distribution (Fig. 6c). To examine the effects of SWELL1 knock-down in vivo during the development of diet-induced obesity we generated adenoassociated viruses (AAV/Rec2) for sustained viral expression of shSWELL1, as this AAV/ Rec2 serotype has a high tropism for adipose tissue and can sustain expression for up to 12 months ${ }^{49}$. After direct iWAT injection with either AAV/Rec2-shSWELL1-mCherry or AAV/ Rec2-shSCR-mCherry, both groups of mice were placed on a HFD for 16 weeks prior to analysis. Patch-clamp recordings from hypertrophic AAV/Rec2-shSWELL1-mCherry transduced iWAT adipocytes confirm strong SWELL1 silencing compared to AAV/Rec2shSCR-mCherry at 16 weeks post injection (Fig. 6d\&e) and this is associated with reduced adipocyte size (Fig. 6f\&g), as observed with acute adenoviral SWELL1 knock-down in iWAT of obese mice (Fig. 6a-c). Interestingly, while AAV/Rec2 iWAT transduction is patchy and restricted to the injection site (Supplementary Fig. 6a, iWAT), we note wide-spread viral transduction of much of the visceral epididymal white adipose tissue (Supplementary Fig. 6a, eWAT). In addition, we observe transduction of liver and skeletal muscle (Supplementary Fig. 6b), resulting in a pattern of AAV/Rec2 transduction, involving several insulin-sensitive tissues. Nuclear Magnetic Resonance (NMR) measurements reveal a trend toward reduced total \%fat (Supplementary Fig. 6c), accompanied by a small increase in \%lean mass (Supplementary Fig. 6d). As visceral adipose was preferentially transduced by AAV/Rec2 we speculated that the reduction in global adiposity measured by NMR might be driven by contraction of the visceral depot. To discriminate subcutaneous from visceral adipose tissue non-invasively in vivo, we turned to microCT imaging ${ }^{50}$. This revealed a significant reduction specifically in visceral adiposity, with a marked reduction of the epididymal fat depot (Fig. 6h-j), consistent with preferential SWELL1 KD in visceral fat. MicroCT derived 3D reconstructions of visceral adipose of representative AAV/Rec2-shSCR/shSWELL1mCherry are shown in Fig. 6h (right panels, red) and in Movies S1 and S2, respectively. We next examined both glucose and insulin tolerance in these mice, and found that SWELL1 knock down in vivo is associated with impaired glucose tolerance (Fig. 6k) and insulin resistance (Fig. 61).

\section{Adipocyte-restricted SWELL1 deletion induces systemic glucose intolerance and insulin- resistance}

As a complementary approach to the AAV/Rec2-mediated SWELL1 KD experiments we generated adipose-targeted $S W E L L 1$ knock-out (Adipo KO) mice by crossing $S W E L L I^{\mathrm{fl}}$ mice with Adiponectin-Cre mice to achieve adipose-restricted Cre-mediated SWELL1 recombination using the Cre-loxP system ${ }^{51}$ (Fig. 7a). Adipo KO mice show clear adiposerestricted SWELL1 Exon 3 deletion in subcutaneous (iWAT), visceral (eWAT) and brown (BAT) adipose tissue without evidence of recombination in other tissues (Fig. 7a). SWELL1LRRC8 currents are entirely ablated in mature Adipo KO adipocytes compared to WT adipocytes (Fig. 7b\&c). At baseline, Adipo KO mice are indistinguishable from littermate $S W E L L I^{\mathrm{fl}}$ controls with respect to total body weight (Fig. 7d) and adiposity - as assessed by both NMR (Fig. 7e\&f) and iWAT/eWAT weights (Fig. 7g\&h). Moreover, there are no differences in adipocyte size (Supplementary Fig. 7a-f). mRNA expression levels of adipocyte differentiation markers, including Adiponectin, C/EBPa, C/EBP $\beta$, and PPAR $\gamma$ are either unchanged or slightly increased in iWAT of Adipo KO mice compared to WT (Supplementary Fig. 7g). Despite normal adipocyte development, Adipo KO mice exhibit 
impaired glucose tolerance (Fig. 7i) and insulin-sensitivity (Fig. 7j) compared to WT, as observed with systemic AAV/Rec2-mediated SWELL1 knock down (Fig. 6k, i). Collectively, these data indicate that adipocyte SWELL1 is required for normal systemic glycemia and insulin-sensitivity but is dispensable for normal postnatal development and differentiation of adipocytes in vivo under basal conditions.

\section{Adipocyte-restricted SWELL1 ablation limits adiposity and adipocyte size in obese mice}

As SWELL1-LRRC8 activity is augmented in the setting of obesity in mice and humans we next asked whether SWELL1 is required for adipocyte hypertrophy under conditions of over-nutrition. Adipo KO mice placed on a HFD (16 weeks) gain less weight (Fig. 8a), despite no consistent differences in daily food consumption (Fig. 8b), and this is attributed to a marked reduction in percent adiposity (Fig. 8c) and total adipose mass (Fig. 8d); while percent lean mass is proportionately increased (Fig. 8e), with total lean mass unchanged (Fig. 8f). Inguinal and epididymal fat pads are also significantly smaller in Adipo KO mice relative to WT littermate controls (Fig. 8g).

As the reduction in total adiposity and fat pad size observed in Adipo KO mice may arise from fewer adipocytes, smaller adipocytes, or both, we next measured adipocyte size in WT and Adipo KO mice. Measurements of adipocyte cross-sectional areas from $\mathrm{H} \& \mathrm{E}$ stained sections of HFD Adipo KO and WT iWAT (Fig. 8h) reveal a striking reduction in adipocyte size in Adipo KO mice relative to WT (Fig. 8i\&j). Overlaying the adipocyte size distribution of lean/obese, WT and Adipo KO mice shows that SWELL1 deletion has no effect on adipocyte size in lean mice but induces a clear leftward shift in adipocyte size in the setting of obesity toward a lean phenotype (Fig. 8k). Adipocyte size of freshly dissociated adipocytes isolated from HFD Adipo KO iWAT are also significantly smaller than WT (Supplementary Fig. 8a\&b), with a leftward shifted size distribution (Supplementary Fig. 8c); comparable in relative size reduction to our viral knock-down studies (Fig. 6b-c \& f-g). Moreover, glucose intolerance and insulin-resistance associated with obesity is further exacerbated in Adipo KO mice compared to WT littermates controls (Fig. 81\&m).

To determine whether these differences in adipocyte size in Adipo KO relative to WT mice are related to differences in energy expenditure we measured heat production, $\mathrm{VO}_{2}, \mathrm{VCO}_{2}$, RER, food consumption, activity level and sleep in both lean (Supplementary Fig. 7h-n) and obese mice (Supplementary Fig. 8d-j) by indirect calorimetry. There are no differences in any measured parameter in lean WT compared to Adipo KO mice. In obese mice we observe an increase in energy expenditure in Adipo KO mice (Supplementary Fig. 8d), but this is associated with an increase in activity level (Supplementary Fig. 8h), which may be driving the increased energy expenditure.

Collectively, these data identify $S W E L L 1$ as a swell-activated, volume-sensitive regulator of adipocyte growth, adiposity and insulin signaling and delineates a SWELL1/LRRD-GRB2Cav1-IRS1-PI3K-AKT2 signaling pathway in adipocytes. 


\section{DISCUSSION}

Our data show that the recently identified membrane protein, SWELL1 ${ }^{19,20}$, is a critical component of a prominent volume-sensitive ion channel in adipocytes that is activated in the context of adipocyte hypertrophy in obesity, and is required for adipocyte expansion and insulin-PI3K-AKT2 signaling via a LRRD-mediated GRB2 interaction. These findings link SWELL1, with adipocyte insulin-signaling and provide a putative molecular mechanism for the previously described effects of adipocyte membrane tension on lipogenesis and intracellular signaling ${ }^{1-4,7,9}$. We propose a working model in which SWELL1 is activated by increases in adipocyte volume during adipocyte hypertrophy, and potentiates insulinPI3K-AKT2 signaling via C-terminal LRRD interactions with GRB2-Cav1-IRS1-IR to support insulin-mediated GLUT4 PM translocation, glucose import and lipogenesis. In this model, SWELL1 senses adipocyte volumetric expansion and acts as a feed-forward amplifier to further promote adipocyte expansion, energy storage, and enhance insulinAKT2 signaling during times of caloric excess (feeding). In support of this model, Eduardsen et al. ${ }^{9}$ demonstrated that osmotically-induced increases in cellular volume could potentiate insulin signaling in 3T3-L1 preadipocytes, through unknown mechanisms. Moreover, Chen and Chalfie ${ }^{52}$ recently demonstrated a similar phenomenon in the touch receptor neurons of $C$. elegans, implying that the concept of "mechano-tuning" of insulin signaling may be a universal theme in cell biology.

To add further complexity, SWELL1 is known to form heteromultimers with LRRC8b$\mathrm{e}^{19,28}$, which modifies channel gating, and may influence the diversity of molecular interactions with different protein partners based on the relative abundance of LRRC8b-e in a given cell type or tissue. Therefore, depending on the expression profile of LRRC8 proteins in different tissues, SWELL1 modulation of intracellular signaling may vary in a cell-type dependent fashion. For example, previous studies identified the broadly expressed membrane protein LRRC8c, also named $\underline{\boldsymbol{F}}$ actor of $\underline{\boldsymbol{A}}$ dipocyte $\underline{\boldsymbol{D}}$ ifferentiation $(F A D) 158$, as a factor required for adipocyte differentiation ${ }^{53}$ and diet-induced obesity ${ }^{54}$ - through unidentified molecular mechanisms. As LRRC8c (FAD158) forms a complex with SWELL1 ${ }^{28}$ and modifies SWELL1-LRRC8 channel function ${ }^{19,28}$, our findings may provide a mechanism for FAD158/LRRC8c action in adipocyte biology.

It is intriguing that adipose-targeted $S W E L L 1$ ablation appears not to grossly affect adipocyte development, adipocyte size or adiposity under basal conditions, but results in significant suppression of adipocyte expansion in the setting of obesity. This is in line with our observation that SWELL1 is activated in hypertrophic adipocytes from obese mice and humans, suggesting that SWELL1 activity is primarily required for extremes of adipocyte expansion in obese states. This phenotype is also consistent with the finding that SWELL1 deletion disrupts specifically PI $3 \mathrm{~K}-A K T 2$ signaling - an AKT isoform that is dispensable for adipocyte development ${ }^{22,55}$, and instead is primarily important in "obesogenic"

adipogenesis ${ }^{56}$ and systemic glucose homeostasis 22,55 , in contrast to AKT1 ${ }^{23}$, 57. Moreover, Adipo KO mice phenocopy AKT2 ${ }^{-/-}$mice $^{22,55}$ and adipose-targeted GLUT4 KO mice ${ }^{58}$ with respect to systemic glucose intolerance and insulin resistance, supporting the notion of a SWELL1-regulated insulin-AKT2-GLUT4 pathway. 
In summary, we show that SWELL1 is a required component of a volume-sensitive ion channel complex that is activated in the setting of obesity and positively regulates adipocyte lipid content and glucose uptake via GRB2-mediated modulation of insulin-PI3K-AKT2 signaling. As $S W E L L 1$ is broadly expressed, and PI3K-AKT pathway ubiquitous, and fundamentally intertwined with numerous signaling pathways we anticipate that SWELL1 signaling will be physiologically and pathophysiologically important in a multitude of different tissues and disease states.

\section{METHODS}

\section{Patients}

The University of Iowa Institutional Review Board (IRB) approved the study involving human samples. Informed consent was obtained from human subjects. Visceral adipose tissues were obtained from esophageal fat pads, either from morbidly obese patients who underwent bariatric surgeries or normal control subjects who underwent non-bariatric surgeries at the University of Iowa Hospital. Patients were anonymous to the research team except for information of gender, age, and BMI. Fat tissue was kept in ice cold PBS and transferred to laboratory within 20 minutes.

\section{Animals}

The Institutional Animal Care and Use Committee of the University of Iowa approved all experimental procedures involving animals. Only male mice were used in all studies. The experiments were not randomized. The investigators were blinded to allocation during experiments and outcome assessment for certain experiments: 1. microCT imaging and quantification of adiposity; 2. Nuclear Magnetic Resonance Imaging; 3; glucose tolerance testing; 4. Insulin tolerance testing. All C57BL/6 mice involved in the study were purchased from Charles River Labs. Adiponectin-Cre mice were a kind gift from Dr. Matthew Potthoff at The University of Iowa. All the mice were housed in a temperature, humidity, and light controlled room and allowed free access to water and food. High fat diet was used to induce obesity in mice. Mice were fed at libitum with $60 \% \mathrm{kcal} \%$ high-fat Diet (Research Diets) for $12-16$ weeks starting at the age of $4 \sim 6$ weeks. The weights were recorded weekly after initiation of HFD. Daily food consumption was calculated weekly by monitoring the total food consumption for WT and Adipo KO mice, measured as mean food consumption for 1 to 4 mice caged together.

\section{Adenoviruses and Adeno-associated viruses preparation}

Human adenovirus type 5 with hLRRC8A-shRNA and scrambled non-targeting control was obtained from Vector Biolabs (Ad5-mCherry-U6-hLRRC8A-shRNA, 2.2X10 ${ }^{10} \mathrm{PFU} / \mathrm{ml}$, Ad5-U6-scramble-mCherry, $\left.1 \mathrm{X} 10^{10} \mathrm{PFU} / \mathrm{ml}\right)$. Human adenovirus type 5 with Ad5-CMVCre-mCherry $\left(3 \times 10^{10} \mathrm{PFU} / \mathrm{ml}\right)$ and Ad5-CMV-mCherry $\left(1 \mathrm{X} 10^{10} \mathrm{PFU} / \mathrm{ml}\right)$ were obtained from The University of Iowa Viral Vector Core. The AAV serotype rec2 with hLRRC8AshRNA and mCherry reporter (AAVrec2-mCherry-U6-hLRRC8a, 2.2X10 ${ }^{13}$ vector genomes $(\mathrm{vg}) / \mathrm{ml})$ were produced by Dr. Lei Cao's Laboratory as previously described ${ }^{49}$. Scrambled non-targeting shRNA was used as a control (AAVrec2-mCherry-Scramble, $3.8 \times 10^{13} \mathrm{vg} / \mathrm{ml}$ ). 


\section{Primary mature adipocytes isolation}

Mouse inguinal fat pads were removed and washed with HBSS (Gibco) and then minced. The minced tissue was digested for $30-40$ minutes at $37^{\circ} \mathrm{C}$ in $10 \mathrm{ml}$ of digestion buffer ( $3 \%$ BSA in HBSS, $1 \mathrm{mM} \mathrm{CaCl}_{2}, 1 \mathrm{mM} \mathrm{MgCl} 2,8 \mathrm{mg} / \mathrm{ml}$ collagenase D, 2.4 units $/ \mathrm{ml}$ Dispase II). The digested tissue was filtered through a $220 \mu \mathrm{m}$ mesh, and centrifuged at $1000 \mathrm{rpm}$ for 3 minutes. The supernatant, which contained mature adipocytes, was collected. Cells were washed with DMEM/F12 medium (Sigma) containing 10\%FBS (Gibco), 100 IU penicillin and $100 \mu \mathrm{g} / \mathrm{ml}$ streptomycin by repetitive pelleting. For mature adipocytes, the final adipocyte suspension was aliquoted in a 24-well plate. Glass coverslips coated with Matrigel (Corning) were placed on the surface of the cell suspension upside down, and incubated at $37^{\circ} \mathrm{C}$ for at least $20 \mathrm{~min}$. The coverslips with adipocytes attached were then placed on the bottom of a perfusion chamber for subsequent experiments. Human fat samples were processed using the same protocol.

\section{Mouse primary stromal vascular fraction (SVF) isolation for culture}

$S W E L L 1^{f l}$ mouse inguinal fat pads were prepared and digested as described above. To isolate SVF, the pellet was saved and washed with DMEM/F12 medium (Sigma) containing 10\%FBS (Gibco), $100 \mathrm{IU}$ penicillin and $100 \mu \mathrm{g} / \mathrm{ml}$ streptomycin by repetitive pelleting. SVFs were seeded on collagen-coated plates (BD Bioscience) for subsequent culture and differentiation (see Cell Culture, below).

\section{Cell culture}

No cell lines used in this study were found in the database of commonly misidentified cell lines that is maintained by ICLAC and NCBI Biosample. The cell lines were not authenticated. HEK and 3T3-F442A (Sigma-Aldrich) cells were grown in Dulbecco's Modified Eagle Medium (DMEM) containing $4.5 \mathrm{~g} / \mathrm{L}$ glucose, 10\% FBS (Gibco) and 100 IU penicillin and $100 \mu \mathrm{g} / \mathrm{ml}$ streptomycin. Both cell lines tested negative for mycoplasma. To induce 3T3-F442A cells differentiation, confluent cells were cultured in the above medium supplemented with $5 \mu \mathrm{g} / \mathrm{ml}$ (860 $\mathrm{nM}$ ) insulin (Cell applications, Inc.). To compare lipid content under low glucose condition, DMEM containing $1 \mathrm{~g} / \mathrm{L}$ glucose was used. Primary mouse SVFs were seeded in collagen-coated plates (BD Bioscience) and cultured in a growth medium which consisted of DMEM/F12 medium (Sigma) supplemented with 10\% FBS, $100 \mathrm{IU}$ penicillin and $100 \mu \mathrm{g} / \mathrm{ml}$ streptomycin. When cells reached confluence, adipocytes differentiation was induced by changing the above medium to a differentiation medium. The differentiation medium was the growth medium supplemented with $0.5 \mathrm{M}$ IBMX (Cayman), $1 \mu \mathrm{M}$ Dexamethasone (Cayman), $5 \mu \mathrm{g} / \mathrm{ml}(860 \mathrm{nM}$ ) Insulin and $1 \mu \mathrm{M}$ Rosiglitazone (Enzo). Cells were maintained in the differentiation medium for 10-12 days. For immortalized human preadipocytes (NPAD) ${ }^{24}$, the cells were cultured in Preadipocyte Basal Medium 2 (PBM-2) (Lonza, MD) supplemented with 10\% FBS, L-glutamine, gentamycin, and amphotericin according to the manufacturer's instructions. For differentiation, the culture medium was supplemented with dexamethasone, IBMX, indomethacin, and human insulin prepared according to the manufacturer's instructions (Lonza, MD). Cells were left in the differentiation medium for 10-12 days. 


\section{Adenovirus and adeno-associated virus injections to mouse inguinal adipose depot}

Mice were anesthetized by isofluorane (1-4\%), and placed in a prone position. The skin above the inguinal fat pad was prepared in a sterile fashion and an incision made to expose the inguinal fat pad. Virus diluted in sterile PBS: $1 \times 10^{10} \mathrm{vg} / 20 \mu \mathrm{l}$ (adeno-associated virus) or $1 \times 10^{9} \mathrm{PFU} / 40 \mu \mathrm{l}$ (adenovirus) for lean mouse, $2 \times 10^{9} \mathrm{PFU} / 100 \mu \mathrm{l}$ (adenovirus) for obese mouse was injected at multiple sites of the fat pad using a $0.3 \mathrm{cc}, 31 \mathrm{G}$ insulin syringe. After the injection, incisions were closed with surgical clips.

\section{CRISPR/cas9-mediated gene knockout in 3T3-F442A cells}

Guide RNA sequences targeting SWELL1 gene were designed using web based CRISPR design tool (http://crispr.mit.edu/) (Supplementary Table 4) and were cloned in a Cas9 expressing bicistronic vector (pSpCas9(BB)-2A-Puro) as described ${ }^{59}$. 3T3-F442A cells were either nucleofected with single (399 for KO1) or double (295/399 for KO2) guide vectors using Amaxa ${ }^{\circledR}$ Cell Line Nucleofector ${ }^{\circledR}$ Kit V per manufacturer's instructions. Puromycin selection medium $(1 \mu \mathrm{g} / \mathrm{ml})$ was added 48 hours after nucleofection and maintained for 5 days. The cell pool was expanded and clonally diluted. Genomic modifications of individual clones were confirmed by Sanger sequencing for KO1, whereas a PCR screen was used to confirm the deletion of the gene fragment between the two guide pairs for KO2. Control 3T3-F442A adipocytes were either WT 3T3-F442A adipocytes or 3T3-F442A clonal lines isolated from the cas9/gRNA transfections that did not undergo CRISPR/cas9-mediated gene editing.

\section{Generation of WT/GRB2KD and SWELL1 KO/GRB2 KD 3T3-F442A adipocytes}

Lenti GRB2 and LUC (luciferase) shRNAs, packaging, and envelope plasmids are kind gifts from Dr. Jon C. Houtman at The University of Iowa. The LUC shRNA was used as a control. Viral production and transduction were performed as described in Bilal et. al ${ }^{60}$. In brief, lentiviral vector plasmid, packaging, and envelope plasmids were transfected into 293T cells. Virus containing supernatant was harvested and used to transduce WT and SWELL1 KO 3T3-F442A cells. Cells were incubated with virus in the presence of $8 \mu \mathrm{g} / \mathrm{ml}$ Hexadimethrine bromide (Sigma) for 72 hours. Post transduction, the cells were selected with $0.5 \mu \mathrm{g} / \mathrm{ml}$ puromycin. Puromycin was then gradually increased to $2 \mu \mathrm{g} / \mathrm{ml}(0.5 \mu \mathrm{g} / \mathrm{ml}$ increments). Non-transduced cells were used as selection control. Selection was maintained for an additional week after the selection control cells were 100\% sloughed off. The transduced cells were then cultured and differentiated as described in cell culture.

\section{Generation of CRISPR/cas9 mediated SWELL1 floxed (SWELL1 ${ }^{\text {fl) }}$ ) mice and Adipose- targeted SWELL1 KO mice (Adipo KO mice)}

SWELL1 intronic sequences were obtained from Ensembl Transcript ID ENSMUST00000139454. All CRISPR/Cas9 sites were identified using ZiFit Targeter Version 4.2 (http://zifit.partners.org/ZiFiT/). Sites with the presence of $\mathrm{G}$ and A nucleotides at sgRNA position 1-4 and GC content between $35-75 \%$ were preferred. Sites with potential off-target binding were identified with CRISPR design tool (http://crispr.mit.edu). A full list of potential off targets for each CRISPR-Cas9 target sequence was obtained using CasOT (http://eendb.zfgenetics.org/casot/). Pairs of oligonucleotides corresponding to the chosen 
CRISPR-Cas9 target sites were designed, synthesized, annealed, and cloned into the pX330U6-Chimeric_BB-CBh-hSpCas9 construct (Addgene plasmid \# 42230), following the protocol detailed in Le Cong et al ${ }^{61}$. Oligonucleotides are listed in Supplementary Table 5. ssODNs (Supplementary Table 5) donors were designed to incorporate a LoxP sequence and unique restriction enzyme site at the cut site of the CRISPR-Cas9 target sequence along with $60 \mathrm{bp}$ of homology on either side. CRISPR-Cas9 reagents and ssODNs were injected into the pronuclei of F1 mouse strain embryos at an injection solution concentration of $5 \mathrm{ng} / \mu \mathrm{l}$ and $75-100 \mathrm{ng} / \mu \mathrm{l}$, respectively. Correctly targeted mice were screened by PCR across the predicted loxP insertion sites on either side of Exon 3. In mice that showed the expected shift in amplicon size from ssODN insertion, we subsequently performed restriction enzyme digest on the PCR product to further confirm correct ssODN insertion. Adipo KO mice were then generated by crossing SWELL ${ }^{\mathrm{fl} / \mathrm{fl}}$ mice with Adiponectin-Cre mice to obtain Adiponectin-Cre/SWELL1 ${ }^{\mathrm{fl} / \mathrm{fl}}$ (Adipo KO) mice. These mice were maintained on a C57B6/129 mixed background. Age and gender-matched SWELL1 ${ }^{\mathrm{fl} / \mathrm{fl} l}$ littermates served as controls.

\section{Induction of SWELL1 knockout in adipocytes isolated from SWELL1 ${ }^{\mathrm{fl}}$ mice}

SVFs were isolated from $S W E L L 1^{f l}$ mice as described above, and cultured in DMEM/F12 medium with $10 \%$ FBS. Once cells reach 30-40\% confluency, culture medium was switched to DMEM/F12 with 2\% FBS. Adenovirus was added in the medium and incubated with cells for 6 hours. The cells were then washed with fresh medium three times and cultured in DMEM/F12 medium with 10\% FBS for another 5-7 days before differentiation.

\section{RNA isolation and quantitative RT-PCR}

Total RNA was isolated from either adipocytes or inguinal fat pads of WT/Adipo KO mice solubilized in TRIzol using PureLink ${ }^{\mathrm{TM}}$ RNA kit (Life Technologies). Complementary DNA (cDNA) was synthesized using iScript ${ }^{\mathrm{TM}}$ Reverse Transcriptase kit (BioRad) with $1 \mu \mathrm{g}$ of purified RNA. qRT-PCR reaction was setup using Power SYBR green PCR master mix (Applied Biosystems) containing $0.5 \mu \mathrm{L}$ of cDNA and the amplification curves were monitored in StepOnePlus Real-Time PCR instrument (Applied Biosystems). All measurements were carried out in triplicates and GAPDH was used as internal standard for $\Delta \Delta \mathrm{C}_{\mathrm{T}}$ calculations. The qRT-PCR primers used are listed in Supplementary Table 3.

\section{RNA Sequencing and analysis}

The libraries were prepared using the Illumina TruSeq Stranded mRNA Sample Preparation kit (Illumina) using $500 \mathrm{ng}$ of input total RNA. The molar concentrations of the indexed libraries were measured using the High Sensitivity DNA kit (Agilent Technologies) and combined equally into one pool. The molar concentration of the pool was measured using Illumina Library Quantification Kit (KAPA Biosystems) and sequenced on the HiSeq 2500 sequencer (Illumina) with a 100 bp Paired-End SBS chemistry (Illumina). Paired-end FASTQ files were aligned to the Mus_musculus_GRCm38_Ensembl reference genome with TopHat v8.7on GenePattern. Aligned BAMs were used as input to Cufflinks v2.02 for transcript quantification. FPKM files from Cufflinks were converted to GCT files via the GenePattern module Fpkm_trackingToGct. Differential expression analyses were computed by t-test in R. Corrections for multiple hypotheses were performed with the Benjamini 
Hochberg method. Gene Set Enrichment was performed from the pre-ranked differentially expressed genes (ranked by t-score) using the GSEA Preranked GenePattern module with C2-Biocarta as the gene sets database.

\section{Electrophysiology}

All experiments were conducted at room temperature. Currents were recorded with either an Axopatch 200B amplifier or a MultiClamp 700B amplifier paired to a Digidata 1550 digitizer and both used pClamp 10.4 software. For mechanical swelling, whole-cell recordings were performed in extracellular solution (in $\mathrm{mM}$ ): $135 \mathrm{NaCl}, 5.4 \mathrm{CsCl}, 10$ HEPES, 10 Glucose, $1 \mathrm{CaCl}_{2}, 3 \mathrm{MgCl}_{2}, 0.1 \mathrm{CdCl}_{2}, \mathrm{pH} 7.4$ with $\mathrm{NaOH}$, and positive pressure applied via patch-pipette. In a subset of experiments, a pressure manometer was placed in-line with the patch pipette to quantify the positive pressure applied to achieve mechanical swelling. The intracellular solution contained (in mM): $120 \mathrm{~L}$-aspartic acid, 20 $\mathrm{CsCl}, 1 \mathrm{MgCl}_{2}$, 5 EGTA, 10 HEPES, $5 \mathrm{MgATP}, 120 \mathrm{CsOH}, 0.1 \mathrm{GTP}$, pH 7.2 with $\mathrm{CsOH}$. For hypotonic swelling, extracellular solution consisted of the following (in $\mathrm{mM}$ ): $90 \mathrm{NaCl}$, $2 \mathrm{CsCl}, 1 \mathrm{MgCl}_{2}, 1 \mathrm{CaCl}_{2}, 10$ HEPES, 10 mannitol, pH 7.4 with $\mathrm{NaOH}(210 \mathrm{mOsm} / \mathrm{kg})$. The isotonic extracellular solution consisted of the same composition above but with 110 $\mathrm{mM}$ instead of $10 \mathrm{mM}$ mannitol (300 mOsm $/ \mathrm{kg}$ ). Swell-activated current was elicited by perfusing cells with hypotonic solution $(210 \mathrm{mOsm} / \mathrm{kg})$. The patch electrodes were prepared from borosilicate glass capillaries (WPI) and had a resistance of 2.5-4.8 M $\Omega$ when filled with pipette solution. For perforated patch recordings, the intracellular solution was as above but without ATP and GTP, and contained $360 \mu \mathrm{g} / \mathrm{ml}$ Amphotericin B (Sigma). This approach minimally perturbs the adipocyte plasma membrane, protects against lipid occluding the patch-pipette during recordings, and permits controlled activation of SWELL1-mediated current (via hypotonic swelling) which allows for more accurate quantification than can be achieved using positive-pressure induced adipocyte swelling in whole-cell configuration. The holding potential was $0 \mathrm{mV}$. Voltage steps were elicited from a $0 \mathrm{mV}$ holding potential from -80 to $+80 \mathrm{mV}$ in $20 \mathrm{mV}$ increments every $1 \mathrm{~s}$. Voltage ramps from -100 to $+100 \mathrm{mV}$ (at $0.4 \mathrm{mV} / \mathrm{ms}$ ) were applied every $4 \mathrm{~s}$. Sampling interval was $100 \mu \mathrm{s}$ and filtered at $10 \mathrm{KHz}$. For perforated-patch recordings, cells with a membrane resistance below G $\Omega$ or access resistance above $20 \mathrm{M} \Omega$ were discarded.

\section{Immunoblotting and immunoprecipitation}

For iWAT Western blot, the inguinal fat pads were harvested 10 minutes after the mouse received an i.p. insulin ( 5 unit $/ \mathrm{kg} \mathrm{BW}$ ) injection. Fat tissues were minced and homogenized in cold RIPA buffer (150 mM NaCl, $20 \mathrm{mM}$ HEPES, $1 \%$ NP-40, 5mM EDTA, PH 7.4) with added proteinase/phosphatase inhibitors (Roche).

For cultured 3T3-F442A and primary adipocytes, cells were washed twice with ice cold PBS, and lysed with the same lysis buffer as above for $30 \mathrm{~min}$ on ice. Lysates were then further lysed with 2 rounds of 10-20 seconds sonication, and centrifuged at 14,000 $\times \mathrm{g}$ for 15 min at $4{ }^{\circ} \mathrm{C}$. Supernatant was collected, and protein concentration determined using a DC protein assay kit (Bio-rad). For immunoblotting, equal amounts of protein $(20-50 \mu \mathrm{g})$ was boiled with SDS loading buffer, electrophoretically separated in 4-15\% gradient SDSPAGE gel (Bio-Rad) under reducing conditions and transferred to PVDF membranes (Bio- 
Rad). For immunoprecipitation experiments, $1 \mathrm{mg}$ of protein lysate was incubated with the specific antibody at $4^{\circ} \mathrm{C}$ for 4 to 8 hours. $30 \mu \mathrm{l}$ of equilibrated protein $\mathrm{A} / \mathrm{G}$ sepharose beads (GE) were added, followed by an additional $2-4$ hours of incubation at $4^{\circ} \mathrm{C}$. The beads were then washed three times with RIPAbuffer, re-suspended in laemmli buffer (Bio-rad), boiled for 5 minutes, separated by SDS-PAGE gel and then transferred to PVDF membranes. Membranes were blocked with 5\% BSA in TBST buffer $(0.2 \mathrm{M}$ Tris, $1.37 \mathrm{M} \mathrm{NaCl}, 0.2 \%$ Tween 20, $\mathrm{pH}$ 7.4) at room temperature for one hour, then incubated with primary antibody either at room temperature for 2 hours or $4^{\circ} \mathrm{C}$ overnight. Membranes were then incubated with corresponding secondary antibody (Bio-Rad, Goat-anti-rabbit \#170-6515, Goat-antiMouse \#170-5047; abcam, Mouse-anti-Rabbit IgG heavy chain \#ab99702 or mouse-antiRabbit IgG light chain \# ab99697, all used at 1:10000) at room temperature for one hour. The signals were visualized by chemiluminescence (Pierce) and imaged using a ChemiDoc XRS+ imaging system (Bio-rad). Protein band intensities were analyzed using ImageJ software (National Institute of Health). To measure insulin-stimulated GLUT4 translocation, differentiated 3T3-F442A adipocytes were serum starved for 6 hours, and insulin ( 0 and 100 $\mathrm{nM}$ ) stimulated for $15 \mathrm{mins}$. Cells were placed on ice for $10 \mathrm{~min}$ to stop trafficking. The cell surface was labeled for $30 \mathrm{~min}$ on ice with $0.5 \mathrm{mg} / \mathrm{ml}$ EZ-link Sulfo-NHS-Biotin (ThermoPierce) in PBS. After quenching with $100 \mathrm{mM}$ Glycine in PBS, the cells were lysed in the same lysis buffer described above. $1 \mathrm{mg}$ of each protein lysate was diluted to $1 \mu \mathrm{g} / \mu \mathrm{l}$ and incubated with $50 \mu \mathrm{l}$ of NeutrAvidin beads (Thermo-Pierce) for 16 hours at $4{ }^{\circ} \mathrm{C}$ prior. The beads were washed three times with RIPA buffer, re-suspended in $2 \mathrm{X}$ laemmli buffer (Bio$\mathrm{rad}$ ), boiled for 10 minutes, separated by SDS-PAGE gel and Western blot performed as described above. Caveolin-1 (Cav1) served as an internal control to confirm equal PM protein loading between insulin-stimulation conditions.

The primary antibodies used in current study include: from Cell Signaling: anti-phospho Akt1 (\#9018), anti-phospho Akt2 (\#8599), anti-Akt (\#4685), anti-IR (\#3020), anti-Cav1 (\#3267), anti-phospho-AS160 (\#4288), anti GSK-3 $\beta$ (\#9832), anti-phospho-GSK-3 $\beta$ (\#9336), anti-phospho-FoxO1(Thr24)/FoxO3a(Thr32) (\#9464), anti-FoxO1 (\#9462) and anti- $\beta$-Actin (\#8457); from Santa Cruz: Anti-Grb2 (sc-255); from Covance: Anti-HA (MMS-101P); from Millipore: Anti-Glut4 (\#07-1404), and anti-AS160 (\#07-741); and from BD Scientific: Anti-Grb2 (\#610111). Anti-LRRC8a was a kind gift from Dr. Thomas J. Jentsch ${ }^{19}$. All primary antibodies were used at 1:1000, except for Anti-Grb2 (BD) at 1:5000, Anti-LRRC8A at 1:3000.

\section{AdipoRed imaging}

Lipid content was quantified using AdipoRed assay reagent (Lonza). In brief, differentiated cells were washed with PBS (pH7.4) three times, and incubated with AdipoRed solution at room temperature for 10 minutes. Cells were again washed gently with PBS. Fluorescence signal was measured with an excitation wavelength of $485 \mathrm{~nm}$ and an emission wavelength of $572 \mathrm{~nm}$. Images were captured using an ORCA-Flash4.0 HAMAMATSU digital camera C11440 (HAMAMATSU, Japan). Fluorescence intensity quantifications were carried out using Metamorph software (Molecular Devices, Inc). 


\section{Isolated adipocyte size measurement}

For mature adipocyte size measurement, coverslips with adipocytes attached were transferred to a bath chamber on the stage of an inverted microscope (IX73, Olympus) and visualized using a 20x or 40x objective lens (Olympus). Cell images were captured using an ORCA-Flash4.0 HAMAMATSU digital camera C11440 (HAMAMATSU, Japan) and recorded using Metamorph software (Molecular Devices, Inc.). The cross-sectional area was measured as an indicator of the cell size, using Metamorph software.

\section{Glucose Uptake}

3T3-F442A cells incubated in 24-well culture dishes were washed three times with serum free DMEM, then serum starved for 16-18 hours in 5 mM Glucose DMEM and 1\% BSA. Cells were then washed two times with PBS and incubated in glucose free and pyruvate rich (1 mM) DMEM (Sigma D5030-10X1L) for 30 minutes at $37^{\circ} \mathrm{C}$ with $5 \% \mathrm{CO}_{2}$. Cells were

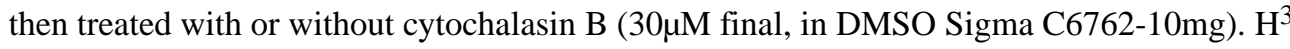
2-Deoxy-D-Glucose (2-DOG) (NET 328A001 mL, Perkin Elmer) was diluted 1:40 in $\mathrm{H}^{1} 2$ DOG (Sigma D6134-5g) then added to cells at a final concentration of $10 \mathrm{mM}$ 2-DOG. Insulin was then added at a final concentration of $100 \mathrm{nM}$ and cells were incubated for 30 minutes at $37{ }^{\circ} \mathrm{C}$ with $5 \% \mathrm{CO}_{2}$. Following incubations cells were washed three times with PBS, and suspended in $500 \mu \mathrm{L} 1 \mathrm{M} \mathrm{NaOH}$ by pipetting up and down. Cell lysates were then freeze thawed twice diluted in Ultima Gold scintillation fluid and analyzed using Beckman Coulter LS-6500 scintillation counter. Protein concentrations were assessed in parallel using BCA analysis. $\mathrm{H}^{3}$ Content was then normalized to $\mathrm{H}^{3}$ 2-DOG standard curves and glucose uptake values were generated normalized to protein content.

\section{Electron microscopy and glycogen estimation}

WT and SWELL1 KO 3T3-F442A adipocytes were fixed using 2.5\% glutaraldehyde in 0.1 $\mathrm{M}$ sodium cacodylate for $30 \mathrm{~min}$ at room temperature (RT). After a rinse with $0.1 \mathrm{M}$ sodium cacodylate, the adipocytes were fixed in a buffer containing $1 \%$ osmium tetroxide and $1.5 \%$ potassium ferrocyanide for $30 \mathrm{~min}$ at RT. Cells were stained with $2.5 \%$ uranyl acetate followed by gradient dehydration with 25 to $100 \%$ ethanol, infiltrated with Eponate 812 before overnight polymerization at $70^{\circ} \mathrm{C}$. Ultrathin sections $(80 \mathrm{~nm})$ were cut using a Leica UC6 ultramicrotome and placed on Formvar and carbon-coated copper grids. After staining with $2.5 \%$ uranyl acetate and Reynold's lead citrate for 2 min each respectively, the grids were viewed under JEOL JEM-1230 transmission electron microscope. ImageJ software was used to calculate the mean glycogen particle size from a total of 1352 particles in five different fields of cell surfaces visualized in two individual grids.

\section{Metabolic phenotyping}

The investigators were blinded to allocation during experiments and outcome assessment for all metabolic phenotyping. For glucose tolerance tests (GTT), mice were fasted for 6 hours. Glucose levels in tail blood were measured with a standard glucometer at the indicated times after an intraperitoneal (IP) injection of D-glucose ( $1 \mathrm{~g} / \mathrm{kg}$ BW for lean mice and AAV virus injected obese mice; $0.75 \mathrm{~g} / \mathrm{kg} \mathrm{BW}$ for HFD mice). For insulin tolerance test (ITT), mice were fasted for 4 hours and blood glucose levels were measured at the above indicated times 
after an IP injection of insulin (HumulinR, 1U/kg BW for lean mice and AAV virus injected obese mice; $1.25 \mathrm{U} / \mathrm{kg} \mathrm{BW}$ for HFD mice). Mouse body composition (fat and lean mass, and water content) was measured by Nuclear Magnetic Resonance (NMR) ${ }^{18}$. Visceral and subcutaneous fat were imaged by Siemen's Inveon PET/CT/SPECT small animal scanner ${ }^{62}$. CT parameters were $220^{\circ}$ degree of rotation, 360 steps, $500 \mathrm{~ms}$ exposure time, $50 \mathrm{kV} /$ 500microamps, binning of 4. Analysis performed on Inveon Research Workplace 4.2.0.15. Metabolic measurements of energy expenditure, respiratory exchange ratio, oxygen consumption, carbon dioxide production, food intake, and physical activity were performed using CLAMS (Comprehensive Lab Animal Monitoring System, Columbus Instruments) by University of Iowa Metabolic Phenotyping Core.

\section{Histological analysis}

Tissues were fixed in $1 \mathrm{X}$ zinc-formalin for $24-48 \mathrm{~h}$ at $4^{\circ} \mathrm{C}$. Paraffin embedding, sectioning, and hematoxylin and eosin staining were performed by University of Iowa Central Microscopy Facility. Images were taken on an Olympus BX-61 light microscope (Olympus, Center Valley, PA) at 20X. Adipocyte area was measured using ImageJ or Metamorph software.

\section{Statistics and Reproducibility}

Statistical tests used are indicated in the figure legends. All statistical tests were performed using GraphPad Prism version 6.02 for Windows (GraphPad Software). No samples or animals were excluded from the analysis. No statistical method was used to predetermine sample size. Neither samples nor animals were randomized. Student's t-test was used for single comparisons, based on the data approximately matching a normal distribution. Wilcoxon or Mann-Whitney test was used as non-parametric test, for data that was not normally distributed. 2-Way ANOVA was used for GTT and ITT analysis. Data are expressed as mean \pm s.e.m., and $P<0.05$ was considered statistically significant. Unless specified, $* \mathrm{p}<0.05,{ }^{* *} \mathrm{p}<0.01, * * * \mathrm{p}<0.001$, and not significant (NS) $\mathrm{p}>0.05$.

\section{Data Availability}

Deep-sequencing (RNA-seq) data that support the findings of this study have been deposited in the Gene Expression Omnibus (GEO) under accession codes GSE86318.

Source data for Fig. 4e-j and Fig. 5g-h have been provided as Supplementary Table 7. All other data supporting the findings of this study are available from the corresponding author on reasonable request.

\section{Supplementary Material}

Refer to Web version on PubMed Central for supplementary material.

\section{Acknowledgments}

We thank Dr. Thomas J. Jentsch (FM/MDC, Berlin) for kindly sharing SWELL1/LRRC8a antibody and Dr. Ardem Patapoutian for sharing Flag-SWELL1 and SWELL $1^{\Delta 91 /+35}$. RNA-Seq data presented herein were obtained at the Genomics Division of the Iowa Institute of Human Genetics. We thank Rita Sigmund, Joe Galbraith, and Michael Knudson of the University of Iowa Tissue Procurement Core facility (TPC) for services provided related to 
acquisition of study specimens (NCI award number P30CA086862) and Susan Walsh of the Small Animal Imaging Core, University of Iowa. We thank the University of Utah Mutation Generation and Detection Core, DNA Sequencing Core, DNA/Peptide Synthesis Core, and Transgenic Gene Targeting Mouse Facility for reagents and services. We thank Drs. Mark Anderson, François Abboud, Peter Snyder, Chris Benson, and Janice Robertson for their thoughtful review of the manuscript. We thank Macaulay Elliot-Hudson and Paul Lüken for assistance with data analysis. This work was supported by grants from the NIH NIDDK 1R01DK106009 (R.S.), the Roy J. Carver Trust (R.S.), the American Heart Association Fellow-to-Faculty Award (R.S.), an American Heart Association Postdoctoral Award (Y.Z.) and an American Cancer Society Pilot Grant (R.S.).

\section{References}

1. Farnier $\mathrm{C}$, et al. Adipocyte functions are modulated by cell size change: potential involvement of an integrin/ERK signalling pathway. Int J Obes Relat Metab Disord. 2003; 27:1178-1186. [PubMed: 14513065]

2. Shoham N, et al. Adipocyte stiffness increases with accumulation of lipid droplets. Biophys J. 2014; 106:1421-1431. [PubMed: 24655518]

3. Shoham N, et al. Static mechanical stretching accelerates lipid production in 3T3-L1 adipocytes by activating the MEK signaling pathway. Am J Physiol Cell Physiol. 2012; 302:C429-441. [PubMed: 22012328]

4. Pellegrinelli V, et al. Human adipocyte function is impacted by mechanical cues. The Journal of pathology. 2014; 233:183-195. [PubMed: 24623048]

5. Salans LB, Knittle JL, Hirsch J. The role of adipose cell size and adipose tissue insulin sensitivity in the carbohydrate intolerance of human obesity. J Clin Invest. 1968; 47:153-165. [PubMed: 16695937]

6. Weyer C, Foley JE, Bogardus C, Tataranni PA, Pratley RE. Enlarged subcutaneous abdominal adipocyte size, but not obesity itself, predicts type II diabetes independent of insulin resistance. Diabetologia. 2000; 43:1498-1506. [PubMed: 11151758]

7. Khan T, et al. Metabolic dysregulation and adipose tissue fibrosis: role of collagen VI. Molecular and cellular biology. 2009; 29:1575-1591. [PubMed: 19114551]

8. Briand N, et al. Caveolin-1 expression and cavin stability regulate caveolae dynamics in adipocyte lipid store fluctuation. Diabetes. 2014; 63:4032-4044. [PubMed: 24969108]

9. Eduardsen K, et al. Cell volume regulation and signaling in 3T3-L1 pre-adipocytes and adipocytes: on the possible roles of caveolae, insulin receptors, FAK and ERK1/2. Cell Physiol Biochem. 2011; 28:1231-1246. [PubMed: 22179011]

10. Oancea E, Wolfe JT, Clapham DE. Functional TRPM7 channels accumulate at the plasma membrane in response to fluid flow. Circ Res. 2006; 98:245-253. [PubMed: 16357306]

11. Numata T, Shimizu T, Okada Y. TRPM7 is a stretch- and swelling-activated cation channel involved in volume regulation in human epithelial cells. Am J Physiol Cell Physiol. 2007; 292:C460-467. [PubMed: 16943238]

12. Muraki K, et al. TRPV2 is a component of osmotically sensitive cation channels in murine aortic myocytes. Circ Res. 2003; 93:829-838. [PubMed: 14512441]

13. Liedtke W, Tobin DM, Bargmann CI, Friedman JM. Mammalian TRPV4 (VR-OAC) directs behavioral responses to osmotic and mechanical stimuli in Caenorhabditis elegans. Proc Natl Acad Sci U S A. 2003; 100(Suppl 2):14531-14536. [PubMed: 14581619]

14. Spassova MA, Hewavitharana T, Xu W, Soboloff J, Gill DL. A common mechanism underlies stretch activation and receptor activation of TRPC6 channels. Proc Natl Acad Sci U S A. 2006; 103:16586-16591. [PubMed: 17056714]

15. Coste B, et al. Piezo proteins are pore-forming subunits of mechanically activated channels. Nature. 2012; 483:176-181. [PubMed: 22343900]

16. Che H, Yue J, Tse HF, Li GR. Functional TRPV and TRPM channels in human preadipocytes. Pflugers Arch. 2014; 466:947-959. [PubMed: 24057349]

17. Sukumar P, et al. Constitutively active TRPC channels of adipocytes confer a mechanism for sensing dietary fatty acids and regulating adiponectin. Circ Res. 2012; 111:191-200. [PubMed: 22668831] 
18. Ye L, et al. TRPV4 is a regulator of adipose oxidative metabolism, inflammation, and energy homeostasis. Cell. 2012; 151:96-110. [PubMed: 23021218]

19. Voss FK, et al. Identification of LRRC8 heteromers as an essential component of the volumeregulated anion channel VRAC. Science. 2014; 344:634-638. [PubMed: 24790029]

20. Qiu Z, et al. SWELL1, a Plasma Membrane Protein, Is an Essential Component of VolumeRegulated Anion Channel. Cell. 2014; 157:447-458. [PubMed: 24725410]

21. Kumar L, et al. Leucine-rich repeat containing 8A (LRRC8A) is essential for T lymphocyte development and function. J Exp Med. 2014; 211:929-942. [PubMed: 24752297]

22. Garofalo RS, et al. Severe diabetes, age-dependent loss of adipose tissue, and mild growth deficiency in mice lacking Akt2/PKB beta. J Clin Invest. 2003; 112:197-208. [PubMed: 12843127]

23. Cho H, Thorvaldsen JL, Chu Q, Feng F, Birnbaum MJ. Akt1/PKBalpha is required for normal growth but dispensable for maintenance of glucose homeostasis in mice. J Biol Chem. 2001; 276:38349-38352. [PubMed: 11533044]

24. Vu BG, Gourronc FA, Bernlohr DA, Schlievert PM, Klingelhutz AJ. Staphylococcal superantigens stimulate immortalized human adipocytes to produce chemokines. PLoS One. 2013; 8:e77988. [PubMed: 24205055]

25. Strange K, Emma F, Jackson PS. Cellular and molecular physiology of volume-sensitive anion channels. Am J Physiol. 1996; 270:C711-730. [PubMed: 8638650]

26. Okada Y, Sato K, Numata T. Pathophysiology and puzzles of the volume-sensitive outwardly rectifying anion channel. J Physiol. 2009; 587:2141-2149. [PubMed: 19171657]

27. Inoue H, Takahashi N, Okada Y, Konishi M. Volume-sensitive outwardly rectifying chloride channel in white adipocytes from normal and diabetic mice. Am J Physiol Cell Physiol. 2010; 298:C900-909. [PubMed: 20107039]

28. Syeda R, et al. LRRC8 Proteins Form Volume-Regulated Anion Channels that Sense Ionic Strength. Cell. 2016; 164:499-511. [PubMed: 26824658]

29. Jinek M, et al. A programmable dual-RNA-guided DNA endonuclease in adaptive bacterial immunity. Science. 2012; 337:816-821. [PubMed: 22745249]

30. Yang H, et al. One-step generation of mice carrying reporter and conditional alleles by CRISPR/ Cas-mediated genome engineering. Cell. 2013; 154:1370-1379. [PubMed: 23992847]

31. Fasshauer M, et al. Essential role of insulin receptor substrate-2 in insulin stimulation of Glut4 translocation and glucose uptake in brown adipocytes. J Biol Chem. 2000; 275:25494-25501. [PubMed: 10829031]

32. Gonzalez E, McGraw TE. Insulin-modulated Akt subcellular localization determines Akt isoformspecific signaling. Proc Natl Acad Sci U S A. 2009; 106:7004-7009. [PubMed: 19372382]

33. Sano H, et al. Insulin-stimulated phosphorylation of a Rab GTPase-activating protein regulates GLUT4 translocation. J Biol Chem. 2003; 278:14599-14602. [PubMed: 12637568]

34. Eguez L, et al. Full intracellular retention of GLUT4 requires AS160 Rab GTPase activating protein. Cell metabolism. 2005; 2:263-272. [PubMed: 16213228]

35. Medina M, Wandosell F. Deconstructing GSK-3: The Fine Regulation of Its Activity. Int J Alzheimers Dis. 2011; 2011:479249. [PubMed: 21629747]

36. Nakae J, et al. The forkhead transcription factor Foxo1 regulates adipocyte differentiation. Dev Cell. 2003; 4:119-129. [PubMed: 12530968]

37. Siddle K. Molecular basis of signaling specificity of insulin and IGF receptors: neglected corners and recent advances. Front Endocrinol (Lausanne). 2012; 3:34. [PubMed: 22649417]

38. Wary KK, Mariotti A, Zurzolo C, Giancotti FG. A requirement for caveolin-1 and associated kinase Fyn in integrin signaling and anchorage-dependent cell growth. Cell. 1998; 94:625-634. [PubMed: 9741627]

39. Pilch PF, Liu L. Fat caves: caveolae, lipid trafficking and lipid metabolism in adipocytes. Trends in endocrinology and metabolism: TEM. 2011; 22:318-324. [PubMed: 21592817]

40. Gustavsson J, et al. Localization of the insulin receptor in caveolae of adipocyte plasma membrane. FASEB J. 1999; 13:1961-1971. [PubMed: 10544179] 
41. Cohen AW, et al. Caveolin-1-deficient mice show insulin resistance and defective insulin receptor protein expression in adipose tissue. Am J Physiol Cell Physiol. 2003; 285:C222-235. [PubMed: $12660144]$

42. Sinha B, et al. Cells respond to mechanical stress by rapid disassembly of caveolae. Cell. 2011; 144:402-413. [PubMed: 21295700]

43. Sedding DG, et al. Caveolin-1 facilitates mechanosensitive protein kinase B (Akt) signaling in vitro and in vivo. Circ Res. 2005; 96:635-642. [PubMed: 15731459]

44. Trouet $\mathrm{D}$, et al. Caveolin-1 modulates the activity of the volume-regulated chloride channel. $\mathrm{J}$ Physiol. 1999; 520(Pt 1):113-119. [PubMed: 10517805]

45. Abascal F, Zardoya R. LRRC8 proteins share a common ancestor with pannexins, and may form hexameric channels involved in cell-cell communication. Bioessays. 2012; 34:551-560. [PubMed: 22532330]

46. Sawada A, et al. A congenital mutation of the novel gene LRRC8 causes agammaglobulinemia in humans. J Clin Invest. 2003; 112:1707-1713. [PubMed: 14660746]

47. Shan X, et al. Suppression of Grb2 expression improved hepatic steatosis, oxidative stress, and apoptosis induced by palmitic acid in vitro partly through insulin signaling alteration. In Vitro Cell Dev Biol Anim. 2013; 49:576-582. [PubMed: 23771793]

48. Liu X, et al. Downregulation of Grb2 contributes to the insulin-sensitizing effect of calorie restriction. American journal of physiology Endocrinology and metabolism. 2009; 296:E10671075. [PubMed: 19240254]

49. Liu X, et al. Adipose tissue insulin receptor knockdown via a new primate-derived hybrid recombinant AAV serotype. Molecular Therapy — Methods \& Clinical Development. 2014; 1

50. Metzinger MN, et al. Correlation of X-ray computed tomography with quantitative nuclear magnetic resonance methods for pre-clinical measurement of adipose and lean tissues in living mice. Sensors (Basel). 2014; 14:18526-18542. [PubMed: 25299952]

51. Lee KY, et al. Lessons on conditional gene targeting in mouse adipose tissue. Diabetes. 2013; 62:864-874. [PubMed: 23321074]

52. Chen X, Chalfie M. Modulation of C. elegans touch sensitivity is integrated at multiple levels. The Journal of neuroscience : the official journal of the Society for Neuroscience. 2014; 34:6522-6536. [PubMed: 24806678]

53. Tominaga $\mathrm{K}$, et al. The novel gene fad158, having a transmembrane domain and leucine-rich repeat, stimulates adipocyte differentiation. J Biol Chem. 2004; 279:34840-34848. [PubMed: 15184384]

54. Hayashi T, et al. Factor for adipocyte differentiation 158 gene disruption prevents the body weight gain and insulin resistance induced by a high-fat diet. Biol Pharm Bull. 2011; 34:1257-1263. [PubMed: 21804215]

55. Cho $\mathrm{H}$, et al. Insulin resistance and a diabetes mellitus-like syndrome in mice lacking the protein kinase Akt2 (PKB beta). Science. 2001; 292:1728-1731. [PubMed: 11387480]

56. Jeffery E, Church CD, Holtrup B, Colman L, Rodeheffer MS. Rapid depot-specific activation of adipocyte precursor cells at the onset of obesity. Nature cell biology. 2015; 17:376-385. [PubMed: 25730471]

57. Chen WS, et al. Growth retardation and increased apoptosis in mice with homozygous disruption of the Akt1 gene. Genes Dev. 2001; 15:2203-2208. [PubMed: 11544177]

58. Abel ED, et al. Adipose-selective targeting of the GLUT4 gene impairs insulin action in muscle and liver. Nature. 2001; 409:729-733. [PubMed: 11217863]

59. Ran FA, et al. Genome engineering using the CRISPR-Cas9 system. Nat Protoc. 2013; 8:22812308. [PubMed: 24157548]

60. Bilal MY, Houtman JC. GRB2 Nucleates T Cell Receptor-Mediated LAT Clusters That Control PLC-gammal Activation and Cytokine Production. Frontiers in immunology. 2015; 6:141. [PubMed: 25870599]

61. Cong L, et al. Multiplex genome engineering using CRISPR/Cas systems. Science. 2013; 339:819823. [PubMed: 23287718] 
62. Lublinsky S, Luu YK, Rubin CT, Judex S. Automated separation of visceral and subcutaneous adiposity in in vivo microcomputed tomographies of mice. J Digit Imaging. 2009; 22:222-231. [PubMed: 18769966] 
a

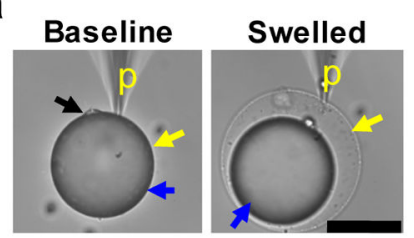

d
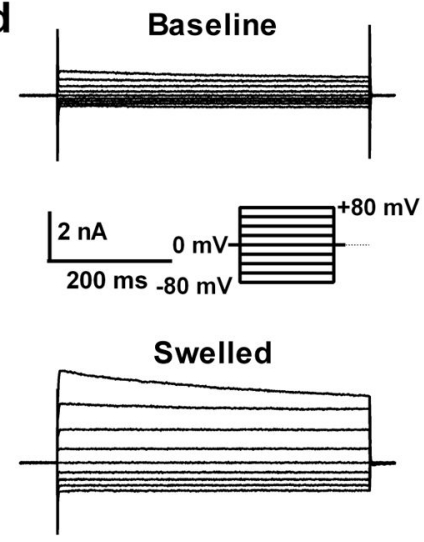

h

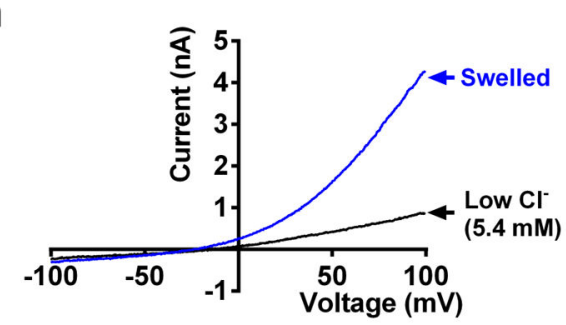

b

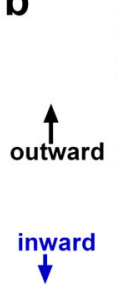

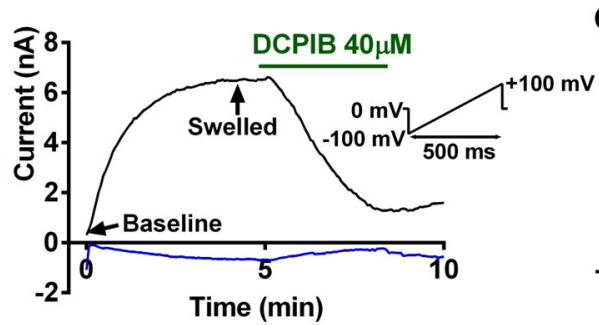

e

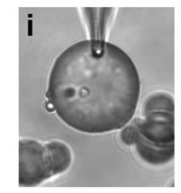

f

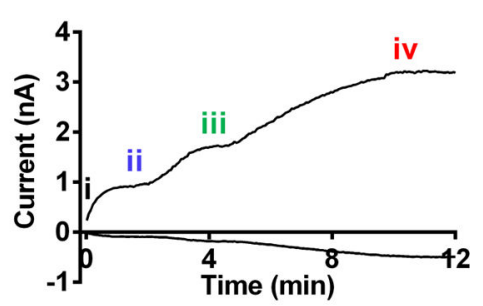

i

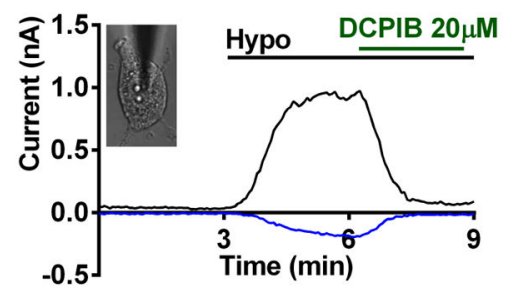

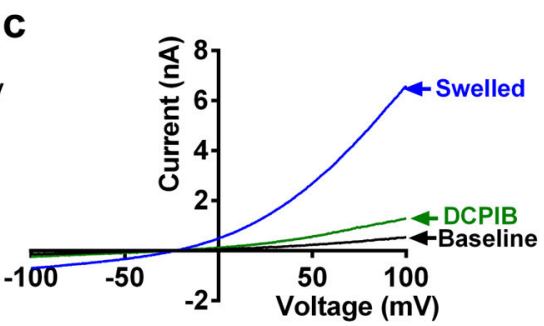

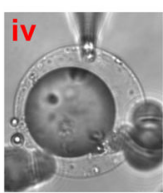

g

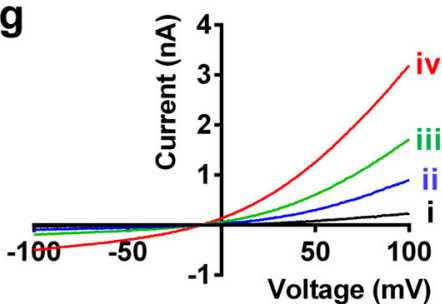

j

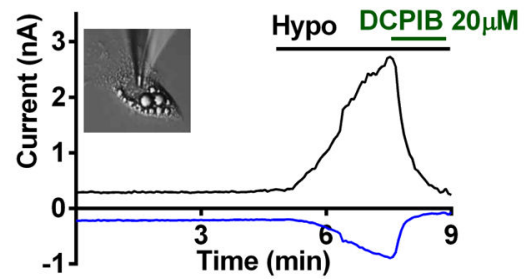

Figure 1. Adipocyte patch clamp reveals prominent swell-activated current in murine and human adipocytes

(a) Mature mouse adipocyte patch-clamped using the whole-cell configuration of the patchclamp technique. Baseline (left) and post swelling (right) by positive pressure via the patch pipette (p). Black arrow: nucleus, yellow arrow: plasma membrane, blue arrow: lipid droplet. (b) Outward and inward swell-activated current over time at, $+100 \mathrm{mV}$ (black) and 7minus; $100 \mathrm{mV}$ (blue), respectively measured in (a) during positive pressure swelling and inhibition by DCPIB. Voltage protocol shown in inset. (c) Current-voltage plots (from b) at baseline (black), after swelling (blue), and with DCPIB inhibition (green). (d) Representative current traces at baseline (top) and after swelling (bottom) during voltage steps (shown in inset). (e) Mature adipocyte swelled to increasing sizes (i-iv). (f) Whole-cell current over time and (g) current-voltage plots at increasing adipocyte sizes, at steady-state (i-iv). e-g $\mathrm{n}=5$ biologically independent experiments. (h) Current-voltage relationship of adipocyte swellactivated current before and after application of low chloride extracellular solution $(5.4 \mathrm{mM}$ $\mathrm{CsCl}$ ). (i-j) Swell-activated current over time in 3T3-F442A adipocyte (i, inset) and in a human adipocyte cell line (j, inset) upon hypotonic swelling (Hypo: $210 \mathrm{mOsm}$ ), followed 
by DCPIB inhibition. Inward current at $-100 \mathrm{mV}$ (negative, blue trace); outward current at $+100 \mathrm{mV}$ (positive, black trace). Scale bar: $50 \mu \mathrm{m}$ for (a). 
a

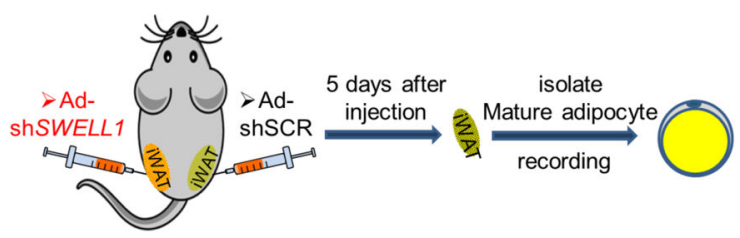

b

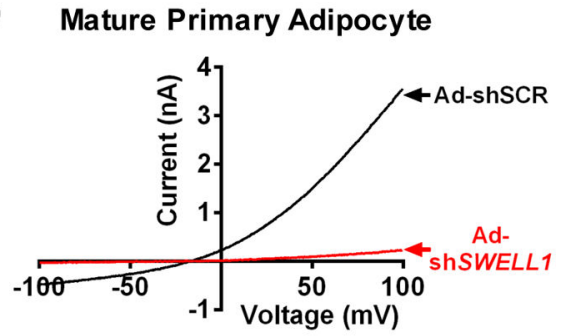

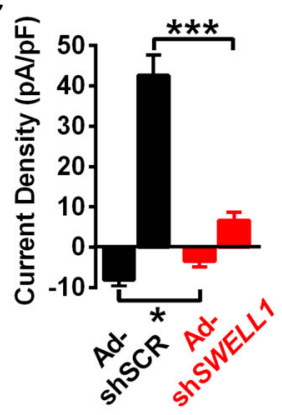

d

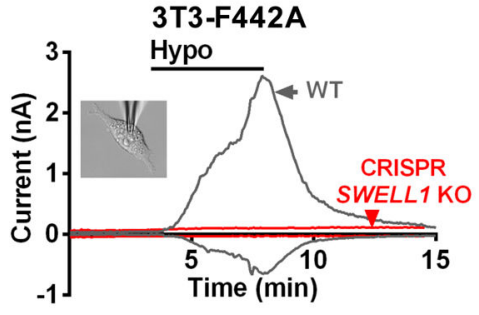

e

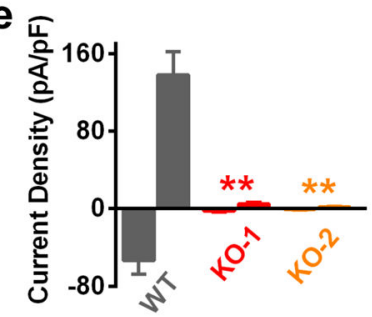

f

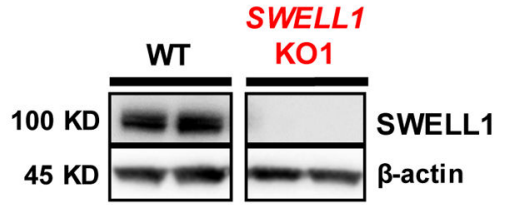

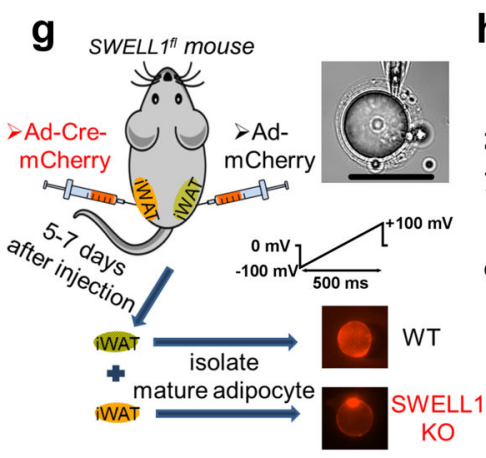

h Mature Primary Adipocyte i Mature Primary Adipocyte j
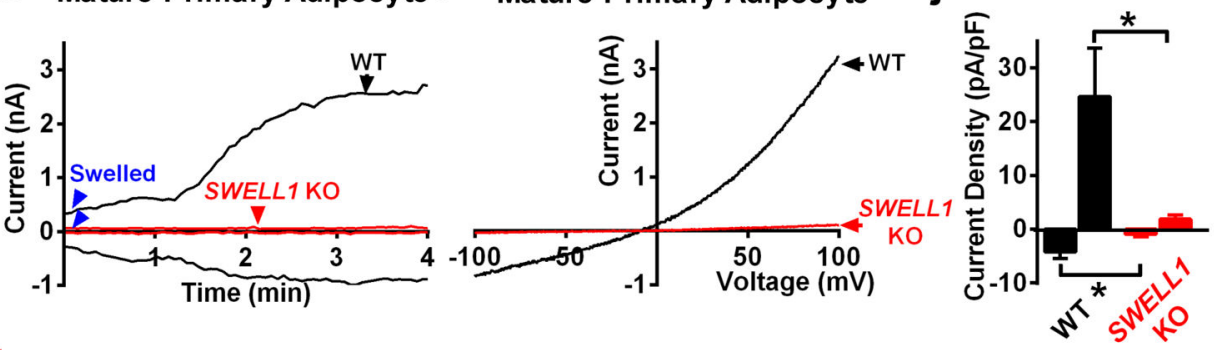

Figure 2. SWELL1 mediates a swell-activated current in adipocytes

(a) Experimental approach for $S W E L L 1$ knock-down, isolation and recording of primary mature mouse adipocytes from iWAT. (b-c) Representative VRAC current-voltage plot (b) and mean outward $(+100 \mathrm{mV})$ and inward $(-100 \mathrm{mV})$ VRAC current density $(\mathbf{c})$ in AdshSCR (black, $\mathrm{n}=6)$ and Ad-sh SWELL1 (red, $\mathrm{n}=6$ ) transduced mature adipocytes upon swell-activation. (d-e) VRAC over time +/- hypotonic swelling in WT (grey trace) and in a CRISPR/cas9 SWELL1 knock out (KO, red trace) 3T3-F442A pre-adipocyte (d) and mean peak outward $(+100 \mathrm{mV})$ and inward $(-100 \mathrm{mV})$ current $(\mathbf{e})$ in WT $\left(\mathrm{n}_{\mathrm{WT}}=12\right)$ and two independent CRISPR/cas9 SWELL1 knock-outs (KO1, KO2) adipocyte cell lines $\left(\mathrm{n}_{\mathrm{KO} 1}=7\right.$, $\mathrm{n}_{\mathrm{KO} 2}=5$ ). (f) Representative Western blot of SWELL1 and $\beta$-actin in WT and CRISPR/cas9 SWELL1 KO 3T3-F442A adipocyte cell line (representative of 20+ blots). Uncropped blots are shown in Supplementary Fig. 9a. (g) Experimental approach for generating SWELL1 knock-out primary mature mouse adipocytes from iWAT of $S W E L L 1^{\mathrm{fl}}$ mice. $(\mathbf{h}-\mathbf{j})$

Representative VRAC current over time (h), current-voltage plot (i), and mean peak outward $(+100 \mathrm{mV})$ and inward $(-100 \mathrm{mV})$ current density $(\mathbf{j})$ induced by swelling (blue arrows) in WT and $S W E L L 1 \mathrm{KO}$ primary mature adipocytes $\left(\mathrm{n}_{\mathrm{WT}}=5, \mathrm{n}_{\mathrm{KO}}=6\right)$. Significance between the indicated groups in $\mathbf{c}, \mathbf{e}$ and $\mathbf{j}$ was calculated using a two-tailed Student's t -test. Exact P- 
values are listed in Supplementary Table 6. Error bars represent mean \pm s.e.m. * $(\mathrm{P}<0.05)$, $* *(\mathrm{P}<0.01), * * *(\mathrm{P}<0.001)$. See also Supplementary Figure 1\&2. Scale bar: $100 \mu \mathrm{m}$ for $(\mathrm{g})$. 
a

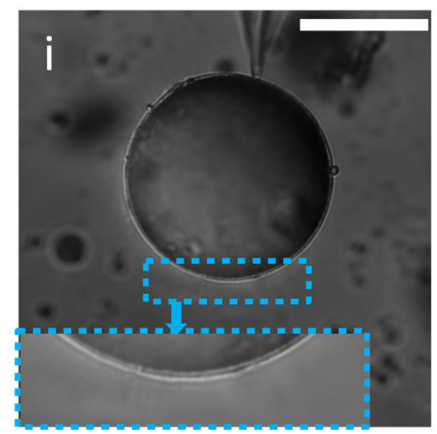

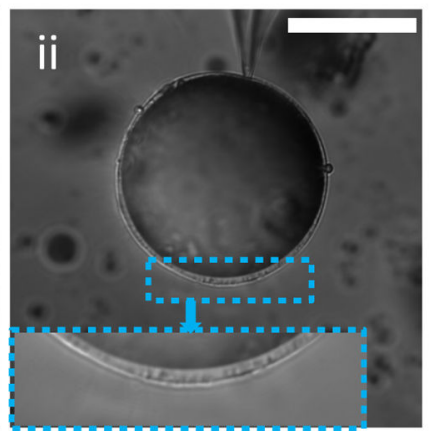

b

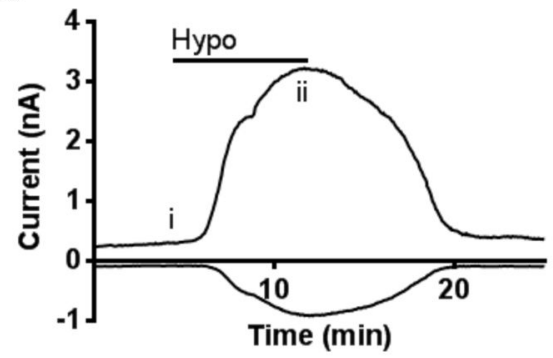

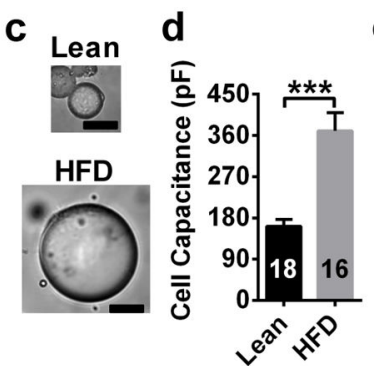

Mature Mouse Adipocyte

f Mature Mouse Adipocyte
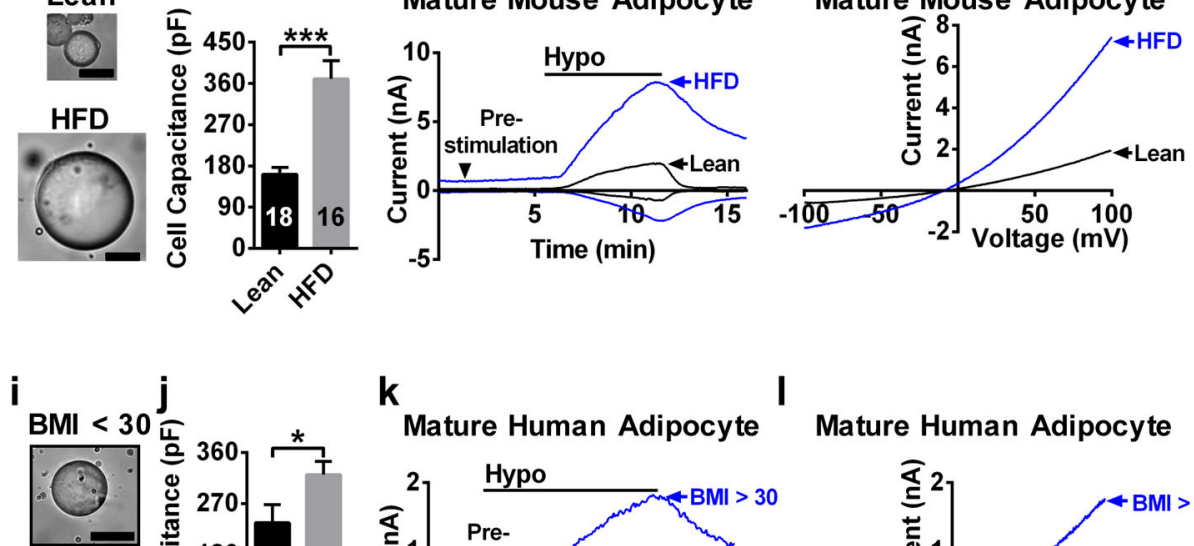

$\mathrm{BMI}>30$
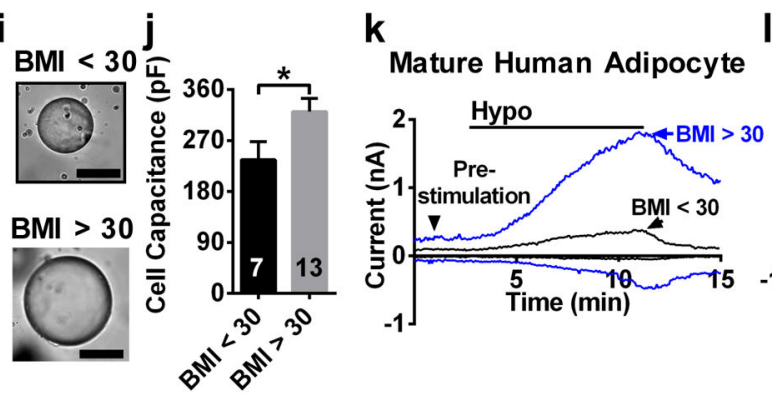

\section{I}
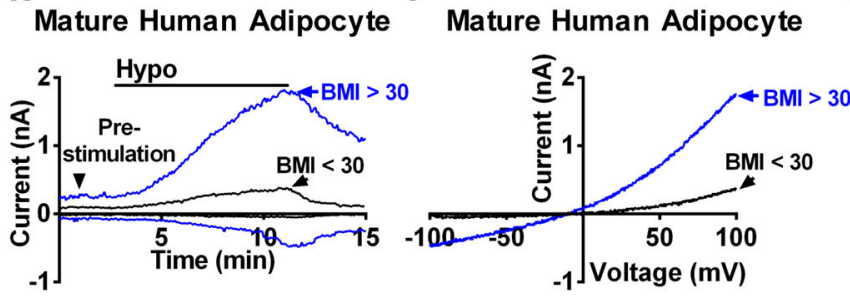
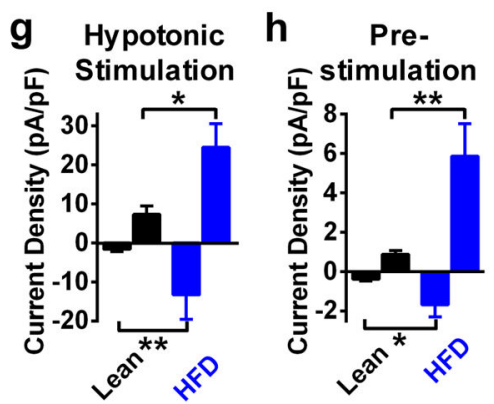

Figure 3. SWELL1-mediated VRAC is increased in adipocytes of obese mice and humans (a) Mature mouse adipocyte before (i) and after (ii) hypotonic swelling under perforatedpatch conditions. Inset shows increase in cytoplasmic volume associated with SWELL1mediated VRAC activation in b. (b) SWELL1-mediated current over time induced by hypotonic swelling (210 mOsm). (c) Images of adipocytes isolated from iWAT of lean mice raised on regular chow and obese mice raised on a HFD. (d) Mean adipocyte capacitance $\left(\mathrm{pF}, \mathrm{n}_{\text {Lean }}=18\right.$ cells, $\mathrm{n}_{\mathrm{HFD}}=16$ cells). (e) SWELL1-mediated current over time +/hypotonic swelling (black triangle: pre-stimulation). (f) SWELL1-mediated current-voltage relationship at peak activation. (g-h) Mean peak outward $(+100 \mathrm{mV})$ and inward $(-100 \mathrm{mV})$ SWELL1-mediated current density after hypotonic swelling $\left(\mathrm{g}, \mathrm{n}=5, \mathrm{n}_{\text {Lean }}=5\right.$ cells, $\mathrm{n}_{\mathrm{HFD}}$ $=5$ cells $)$ and pre-stimulation $\left(\mathbf{h}, \mathrm{n}_{\text {Lean }}=5, \mathrm{n}_{\mathrm{HFD}}=6\right)$ from $\mathbf{e}$. (i) Images of adipocytes isolated from visceral fat of non-bariatric surgery patients $(\mathrm{BMI}<30)$ and obese bariatric surgery patients $(\mathrm{BMI}>30)$. (j) Mean adipocyte capacitance $\left(\mathrm{pF}, \mathrm{n}_{\mathrm{BMI}<30}=7\right.$ cells, $\mathrm{n}_{\mathrm{BMI}>30}$ = 13 cells). (k) SWELL1-mediated VRAC current over time +/- hypotonic swelling (black triangle: pre-stimulation). (l) Current-voltage relationship at peak activation. (m-n) Mean peak outward $(+100 \mathrm{mV})$ and inward $(-100 \mathrm{mV})$ current density after hypotonic activation 
(m) and pre-stimulation (n) (BMI < 30: $\mathrm{n}=7$ cells, BMI > 30: $\mathrm{n}=13$ cells). Significance between the indicated groups in $\mathbf{d}, \mathbf{j}$ were calculated using a two-tailed Student's t-test;

indicated groups in $\mathbf{g}, \mathbf{h}, \mathbf{m}, \mathbf{n}$ were calculated using a two-tailed Mann-Whitney U-test.

Exact P-values are listed in Supplementary Table 6. Error bars represent mean \pm s.e.m. *(P < $0.05)$, **(P < 0.01), ***(P<0.001). Scale bars in $(\mathbf{a}, \mathbf{c}, \mathbf{i})$ are $50 \mu \mathrm{m}$. See also

Supplementary Figure 3. 


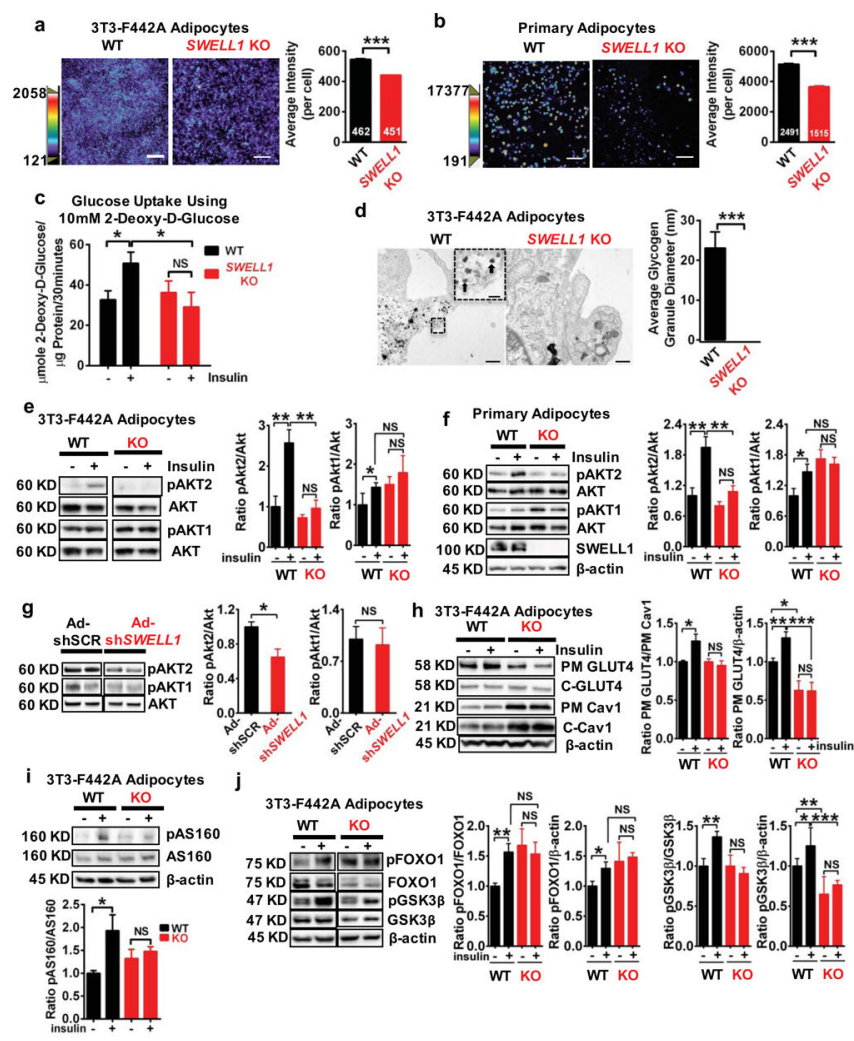

Figure 4. SWELL1 regulates lipid content and glucose metabolism via insulin-pAKT2-AS160GLUT4 and GSK3 $\beta$ signaling

Representative fluorescence images of AdipoRed stained (a) WT and SWELL1 KO 3T3F442A adipocytes and (b) WT and $S W E L L 1$ KO cultured primary adipocytes. Average AdipoRed fluorescence intensity per cell in (a) WT 3T3-F442A $(\mathrm{n}=462)$ and $S W E L L 1 \mathrm{KO}$ 3T3-F442A $(\mathrm{n}=451)$ and $(\mathbf{b}) \mathrm{WT}(\mathrm{n}=2491)$ and $S W E L L 1 \mathrm{KO}(\mathrm{n}=1515)$ cultured primary adipocytes. Measurements pooled from 3 and 4 separate experiments in (a) and (b), respectively. Scale bar: $200 \mu \mathrm{m}$. (c) Insulin-stimulated (100 nM) glucose uptake using 2Deoxy-D-Glucose ( $\mathrm{n}=10$ biologically independent samples per condition). (d) Transmission Electron Microscopy (TEM) images of glycogen granules and mean granule diameter in WT and SWELL1 KO 3T3-F442A adipocytes ( $=5$ fields each). Scale bar: 0.5 $\mu \mathrm{m}$. Inset shows granules (black arrows) in magnified area (Scale bar: $0.1 \mu \mathrm{m})$. Western blots of insulin-stimulated pAKT2, pAKT1, and AKT in (e) WT and SWELL1 KO 3T3F442A adipocytes (0, $100 \mathrm{nM}$ insulin) and (f) WT and $S W E L L 1 \mathrm{KO}$ primary cultured adipocytes $(0,10 \mathrm{nM}) . \mathrm{N}=4$ independent experiments in (e), and $\mathrm{N}=6$ for AKT2 and $\mathrm{N}=7$ for pAKT1 in (f). (g) Western blots of insulin-stimulated pAKT2, pAKT1, and AKT from mouse iWAT injected with Ad-shSCR or Ad-shSWELL1. $\mathrm{N}=4$ independent experiments. (h) Western blots of insulin-stimulated (0, $100 \mathrm{nM})$ plasma membrane (PM) GLUT4, cytosolic (C) GLUT4, PM and C Cav1 and $\beta$-Actin in WT and SWELL1 KO 3T3-F442A adipocytes. Quantification of insulin-stimulated PM GLUT4/PM Cav1 (normalized to unstimulated), PM GLUT4/ $\beta$-actin. N=6 independent experiments. (i,j) Western blots of insulin-stimulated

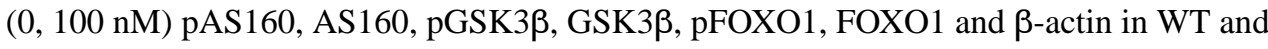
SWELL1 KO 3T3-F442A adipocytes. N=4 independent experiments for pAS160 and N=6 
for pFOXO1 and pGSK3 $\beta$. Significance between the indicated groups in $\mathbf{a}-\mathbf{c}, \mathbf{e}-\mathbf{j}$ were calculated using a two-tailed Student's t-test. Wilcoxon-test used for statistical significance in d. Exact P-values are listed in Supplementary Table 6. Uncropped blots for $\mathbf{e}-\mathbf{j}$ are shown in Supplementary Fig. 9b-p. Statistics source data for $\mathbf{e}-\mathbf{j}$ can be found in Supplementary Table 7. Error bars represent mean \pm s.e.m. $*(\mathrm{P}<0.05), * *(\mathrm{P}<0.01), * * *(\mathrm{P}<0.001)$. See also Supplementary Figure 4. 


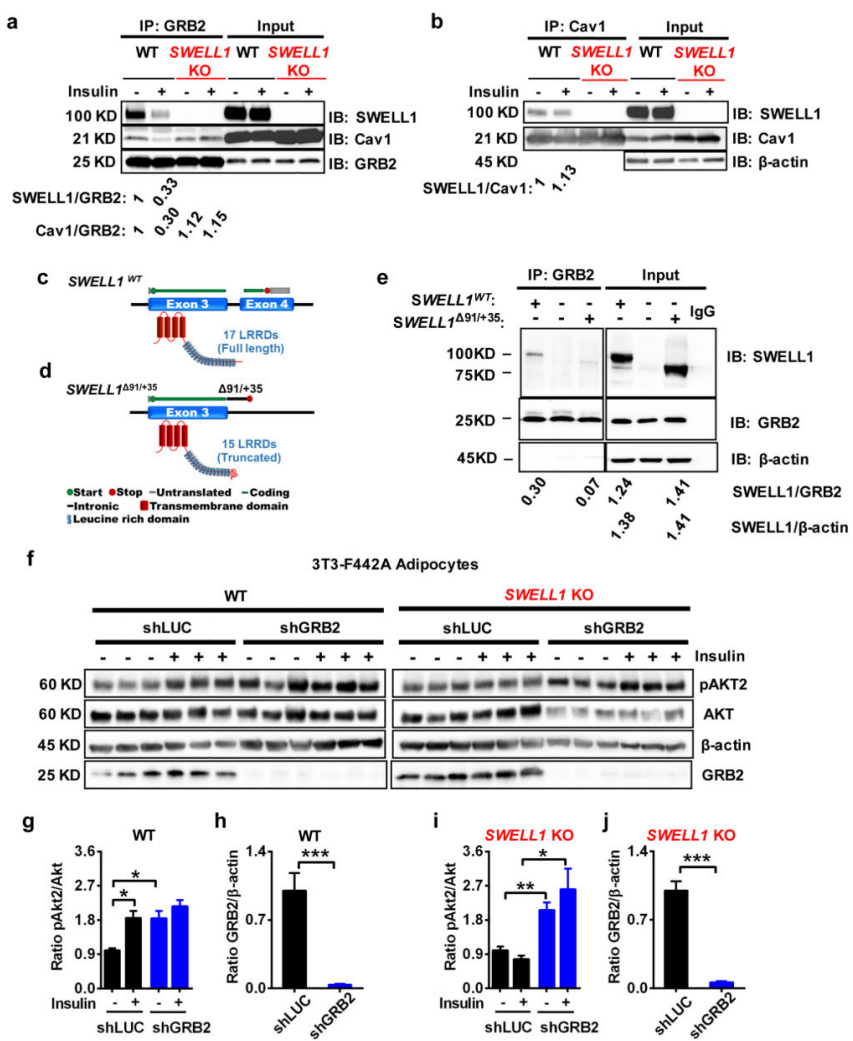

Figure 5. SWELL1/LRRD-GRB2-Cav1-IRS1-IR complex regulates insulin-pAKT2 signaling (a) GRB2 immunoprecipitation from WT and SWELL1 KO 3T3-F442A adipocytes and immunoblot with SWELL1, Cav1 and GRB2 antibodies. Densitometry values for GRB2 coimmunoprecipitated SWELL1 (SWELL1/GRB2) and GRB2 co-immunoprecipitated Cav1 (Cav1/GRB2) over GRB2. Representative blot from 3 independent experiments. (b) Cav1 immunoprecipitation from WT and SWELL1 KO 3T3-F442A adipocytes and immunoblot with SWELL1 and Cav1 antibodies. $\beta$-actin serves as a loading control for input samples. Densitometry values for Cav1 co-immunoprecipitated SWELL1 (SWELL1/Cav1). Representative blot from 3 independent experiments. (c-d) Cartoons depicting $S W E L L 1^{W T}$, and $S W E L L 1^{\triangle 91 /+35}$ mutant. (e) GRB2 immunoprecipitation from HEK cells transfected with $S W E L L 1^{W T}$, non-transfected or $S W E L L 1^{\triangle 91 /+35}$ and then immunoblot with SWELL1, GRB2 antibodies. $\beta$-actin serves as a loading control for input samples. Representative blot from 5 independent experiments. (f) Western blots of pAKT2, AKT, $\beta$-actin, and GRB2 in non-insulin (- : $0 \mathrm{nM})$ and insulin-stimulated (+ : $100 \mathrm{nM}) \mathrm{WT}(\operatorname{shLUC}), \mathrm{WT} / \mathrm{GRB} 2 \mathrm{KD}$ (shGRB2), SWELL1 KO (shLUC) and SWELL1 KO/GRB2 KD (shGRB2) 3T3 F442A adipocytes. (g-j) Densitometry ratio of pAKT2/AKT and GRB2/ $\beta$-actin in WT (g\&h) and $S W E L L 1 \mathrm{KO}$ (h\&i) 3T3-F442A adipocytes. Mean data and statistics from 3 independent experiments shown. Significance between the indicated groups in $\mathbf{g}-\mathbf{j}$ were calculated using a two-tailed Student's t-test. Exact P-values are listed in Supplementary Table 6. Uncropped blots for $\mathbf{a}$ are shown in Supplementary Fig. 9q-s; for b in Supplementary Fig. 9u-w; for e in Supplementary Fig. 9x-z; for $\mathbf{f}$ in Supplementary Fig. 9aa. Statistics source data for $\mathbf{g}-\mathbf{h}$ 
can be found in Supplementary Table 7. Error bars represent mean \pm s.e.m. $*(\mathrm{P}<0.05)$, $* *(\mathrm{P}<0.01), * * *(\mathrm{P}<0.001)$. See also Supplementary Figure 5. 
a

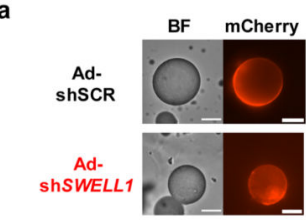

h
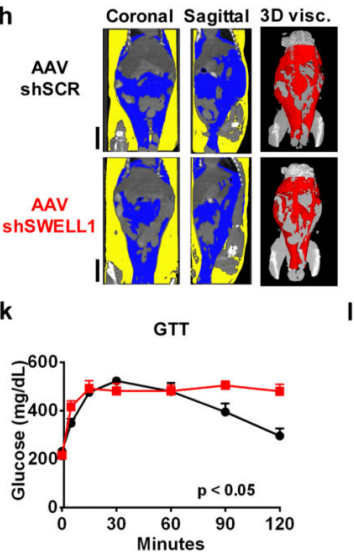

d

Mature Mouse Adipocyte

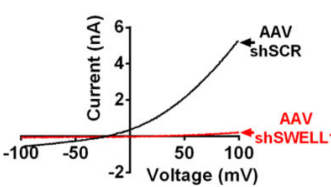

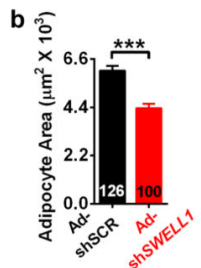
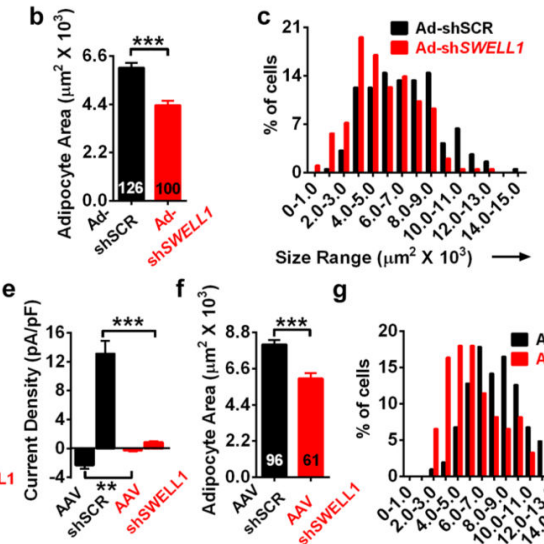

i
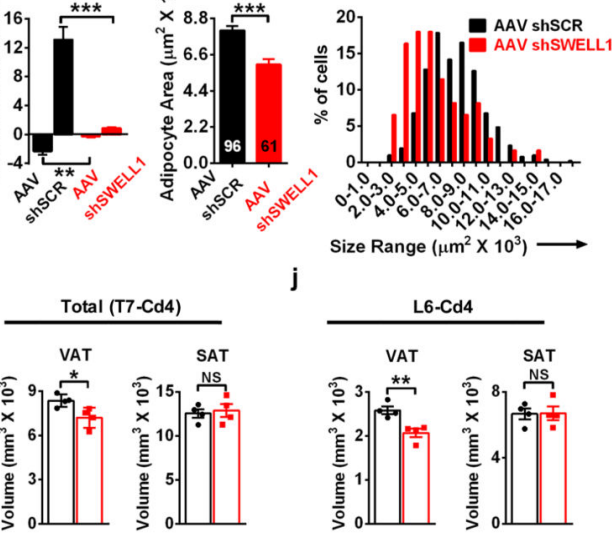

ITT

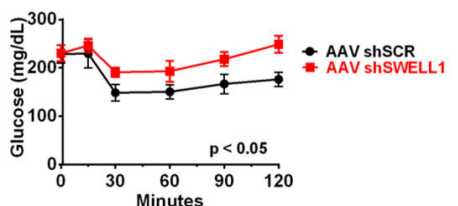

Figure 6. SWELL1 knock-down reduces adipocyte size and adiposity in obese mice (a) Bright field (BF, left) and mCherry fluorescence (right) images of mature adipocytes isolated from iWAT of obese mice (60\% HFD for 12 weeks) 12 days after adenoviral transduction with either Ad-shSCR-mCherry or Ad-shSWELL1-mCherry. Scale bar: $50 \mu \mathrm{m}$. (b-c) Mean adipocyte area (b) and size distribution (c) of Ad-shSCR-mCherry (black bars, $\mathrm{n}$ = 126) and Ad-shSWELL1-mCherry (red bars, $\mathrm{n}=100$ ) transduced adipocytes from iWAT of obese mice. Measurements pooled from 3 Ad-shSCR and 4 Ad-shSWELL1 injected mice (d-e) Current-voltage plots $(\mathbf{d})$ and mean outward $(+100 \mathrm{mV})$ and inward $(-100 \mathrm{mV})$ SWELL1-mediated current (e) induced by positive-pressure swelling of mature primary adipocytes freshly isolated from iWAT of AAV-shSCR $(\mathrm{n}=7)$ and AAV-shSWELL1 $(\mathrm{n}=5)$ transduced mice. $(\mathbf{f}-\mathbf{g})$ Mean adipocyte area (f) and size distribution (g) of AAV-shSCR (black bars, $\mathrm{n}=96$ ) and AAV-sh SWELL1 (red bars, $\mathrm{n}=61$ ) transduced iWAT adipocytes from mice raised on HFD. Measurements pooled from 3 independent experiments. (h) Representative microCT images from the $7^{\text {th }}$ thoracic vertebrae (T7) to $4^{\text {th }}$ tail vertebrae (Cd4) of AAV-shSCR (Top) and AAV-shSWELL1 (Bottom) transduced mice. Left and Center: Coronal and Sagittal 2D sections showing subcutaneous (SAT, yellow) and visceral (VAT, blue) adipose tissue. Right: 3D rendering of visceral adipose tissue only (red). Scale bar: $10 \mathrm{~mm}$. (i-j) Mean adipose tissue volumes of VAT (left) and SAT (right) between anatomic landmarks T7 and Cd4 (i) and L6-Cd4 (j) in AAV-shSCR and AAV-shSWELL1 transduced mice $(\mathrm{n}=4$ each). (k) Glucose tolerance test (AAV-shSCR, $\mathrm{n}=5$ mice; AAV$\mathrm{sh} S W E L L 1 \mathrm{n}=6$ mice). (l) Insulin tolerance test (AAV-shSCR, $\mathrm{n}=6$ mice; AAV- 
$\operatorname{sh} S W E L L 1, \mathrm{n}=5$ mice). Significance between the indicated groups in $\mathbf{b}, \mathbf{e}, \mathbf{f}, \mathbf{i}, \mathbf{j}$ were calculated using a two-tailed Student's t-test, and $\mathbf{k}, \mathbf{I}$ calculated using a 2-way Anova. Exact $\mathrm{P}$-values are listed in Supplementary Table 6. Error bars represent mean \pm s.e.m. * $(\mathrm{P}<$ $0.05), * *(\mathrm{P}<0.01), * * *(\mathrm{P}<0.001)$. See also Supplementary Figure 6. 
a

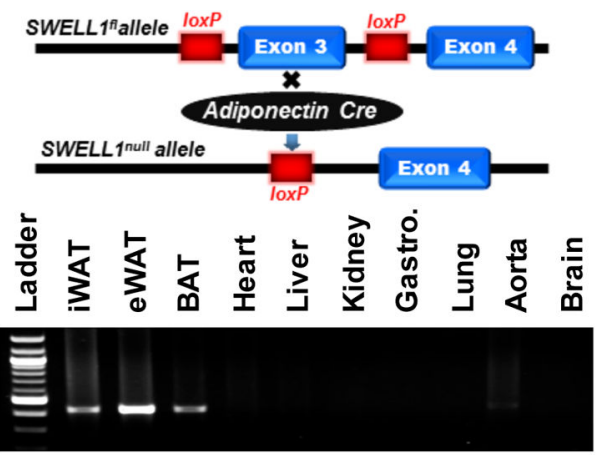

d

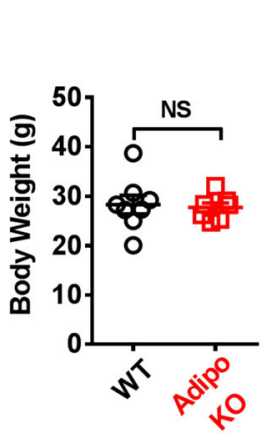

e

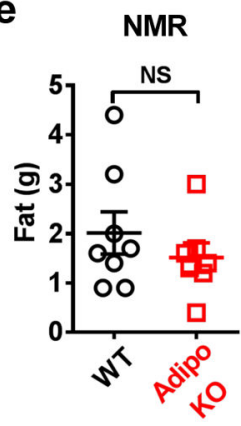

$\mathbf{f}$ b

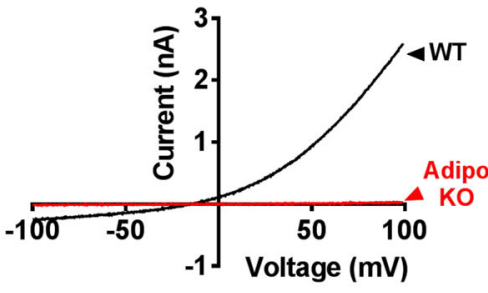

C

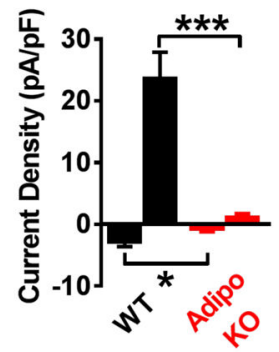

i

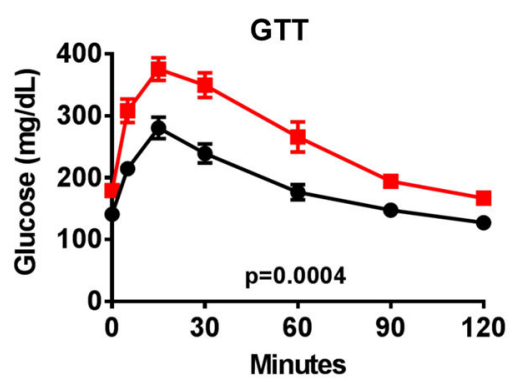

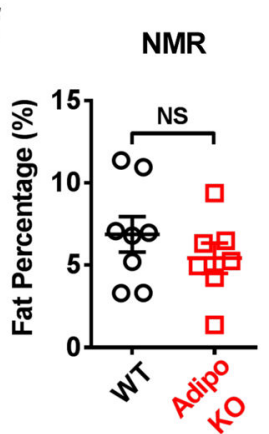

g

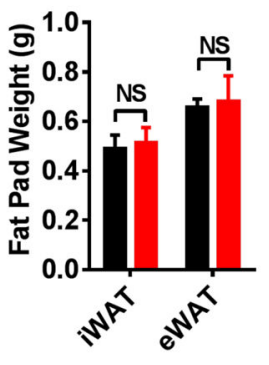

h
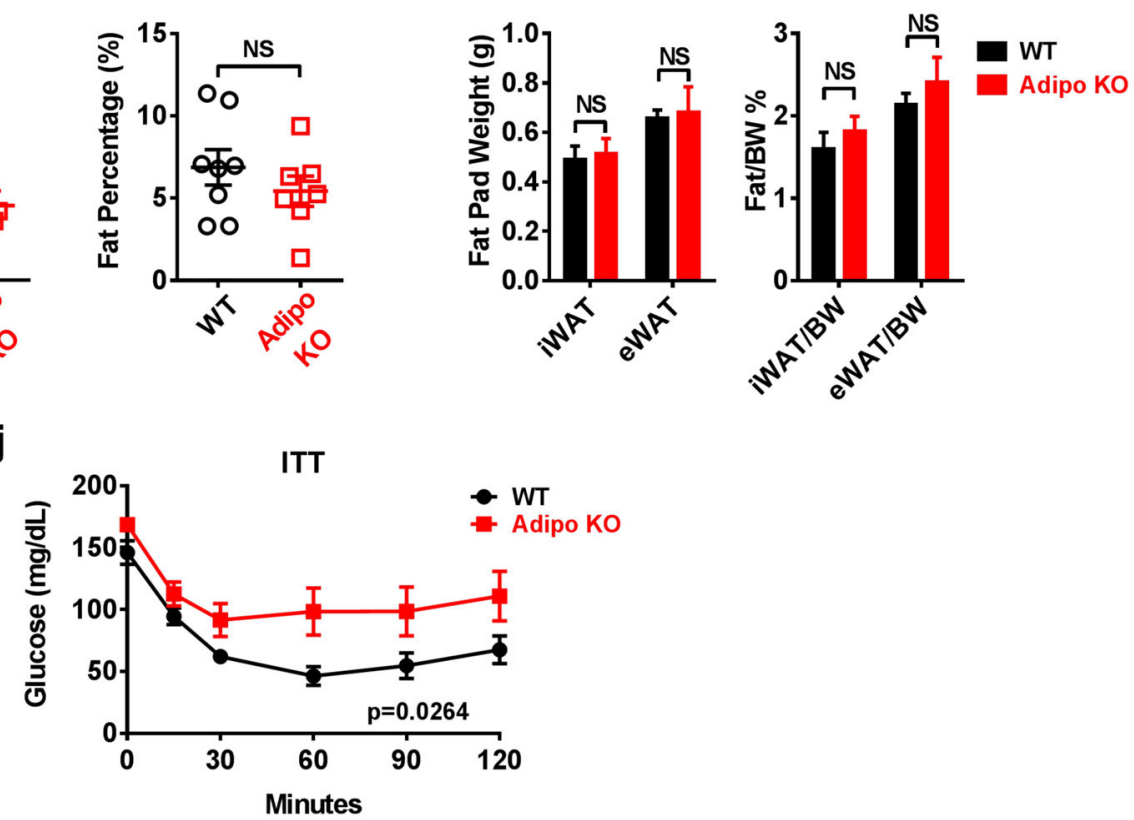

Figure 7. Adipocyte SWELL1 is dispensable for adipose development but required for glucose homeostasis and insulin-sensitivity in vivo

(a) Adipose tissue specific $S W E L L 1$ knockout mice were generated by crossing $S W E L L 1^{f 1}$ to Adiponectin-Cre mice (top). PCR across $S W E L L 1$ Exon 3 in genomic DNA extracted from different tissues of an Adipo KO mouse. Cre-mediated $S W E L L 1$ recombination results in a 426 bp amplicon (bottom). Uncropped blot shown in Supplementary Fig. 9ab. (b-c)

Representative SWELL1-mediated VRAC current-voltage plot (b) and mean outward (+100 $\mathrm{mV})$ and inward $(-100 \mathrm{mV})$ current $(\mathbf{c})$ induced by positive-pressure swelling of primary, mature adipocytes freshly isolated from iWAT of littermate $S W E L L 1^{\mathrm{fl}}(\mathrm{WT})$ control $(\mathrm{n}=5)$ and Adipo KO $(\mathrm{n}=5)$ mice. (d) Body weight of 12 week old $(\mathrm{WT})(\mathrm{n}=8)$ and Adipo KO (n $=7)$ mice raised on regular chow diet. $(\mathbf{e}-\mathbf{f})$ NMR measurements of fat mass (e) and percent fat (f) in WT $(\mathrm{n}=8)$ and Adipo KO $(\mathrm{n}=7)$ mice from $\mathbf{d}$. $(\mathbf{g}-\mathbf{h})$ Total fat pad weights and ratio of fat pad over body weight of iWAT and eWAT in WT and Adipo KO mice $(\mathrm{n}=4$ mice in each group). (i) Glucose tolerance test of WT $(n=9)$ and Adipo KO $(n=8)$ mice raised on regular chow diet. (j) Insulin tolerance test of WT $(n=9)$ and Adipo KO $(n=8)$ mice raised on regular chow diet. Data obtained from 2 separate cohorts of mice. Significance 
between the indicated groups in $\mathbf{c}, \mathbf{d}-\mathbf{h}$ were calculated using a two-tailed Student's t-test, and $\mathbf{i}, \mathbf{j}$ calculated using a 2-way Anova. Exact P-values are listed in Supplementary Table 6. Error bars represent mean \pm s.e.m. $* * *(\mathrm{P}<0.001)$. See also Supplementary Figure 7. 

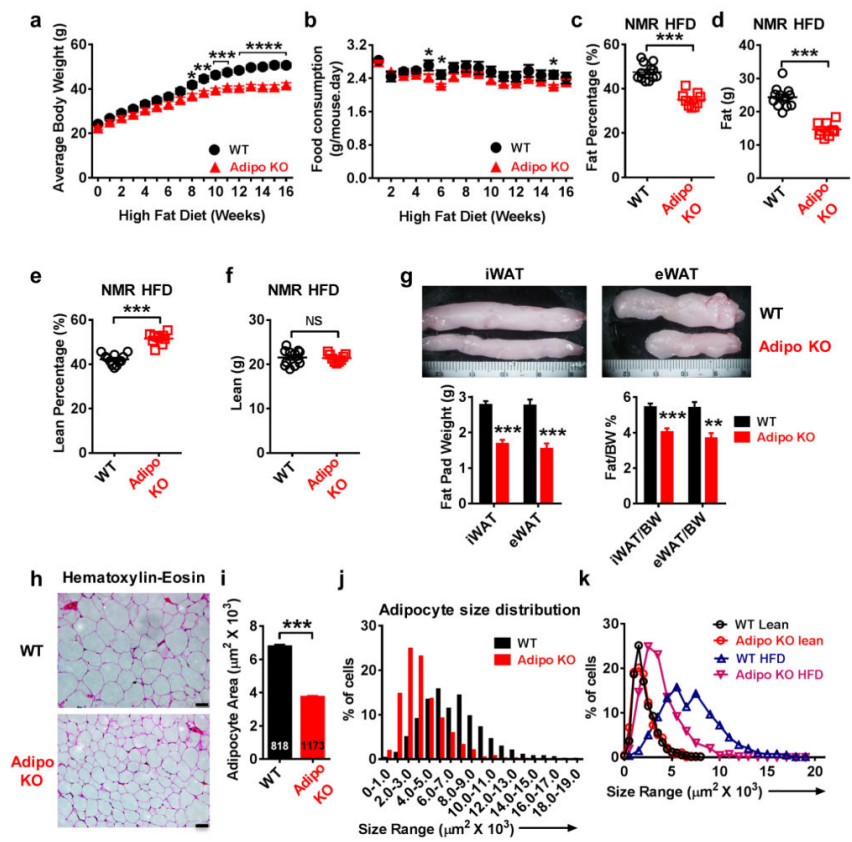

j
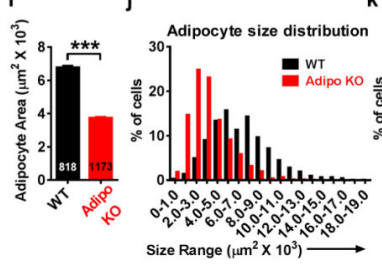

k
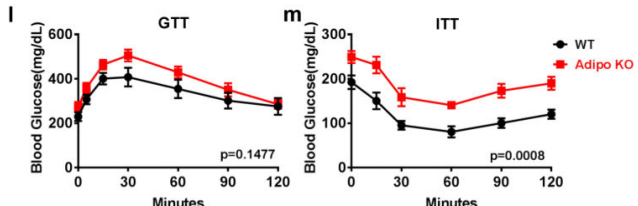

Figure 8. Adipose-restricted SWELL1 deletion limits adipocyte size in obese mice (a-b). Body weight (a) and food consumption (b) in WT $(n=12)$ and Adipo KO $(n=9)$ mice measured at the indicated times on HFD. (c-d) NMR measurements of percent fat (c) and fat mass $(\mathbf{d})$ in WT $(\mathrm{n}=12)$ and Adipo KO $(\mathrm{n}=9)$ mice (HFD for 15 weeks). (e-f) NMR measurements of percent lean mass $(\mathbf{e})$ and lean mass $(\mathbf{f})$ in WT $(n=12)$ and Adipo KO ( $=9$ ) mice (HFD for 15 weeks). (g) Representative inguinal (iWAT) and epididymal (eWAT) fat pads dissected from WT and Adipo KO mice (top). Total fat pad weights and ratio of fat pad over body weight of iWAT and eWAT in WT and Adipo KO mice ( $\mathrm{n}=7$ mice in each group). (h) H\&E staining of iWAT adipose tissue from WT and Adipo KO mice raised on HFD for 17 weeks. $(\mathbf{i}-\mathbf{j})$ Mean WT $(n=818)$ and Adipo KO $(n=1173)$ adipocyte cross-sectional areas (i) and size distribution (j) measured from iWAT histological sections (h). Measurements pooled from 3 separate experiments. (k) Cross-sectional area distribution of adipocytes from H\&E stained sections from WT and Adipo KO mice raised on a lean diet or HFD (17 weeks). (l) Glucose tolerance test and (m) Insulin tolerance test of WT (n=8) and Adipo KO $(\mathrm{n}=7)$ mice raised on HFD for 12 weeks. Scale bars in $(\mathbf{h}): 50 \mu \mathrm{m}$. Data obtained from 2 separate cohorts of mice. Significance between the indicated groups in $\mathbf{a}-\mathbf{j}$ were calculated using a two-tailed Student's t-test, and $\mathbf{l}, \mathbf{m}$ calculated using a 2-way Anova. Exact P-values are listed in Supplementary Table 6. Error bars represent mean \pm s.e.m. * (P $<0.05), * *(\mathrm{P}<0.01)$, *** $(\mathrm{P}<0.001)$. See also Supplementary Figure 8. 